A. Adolphson and S. Sperber

Nagoya Math. J.

Vol. 146 (1997), 55-81

\title{
ON TWISTED DE RHAM COHOMOLOGY
}

\author{
ALAN ADOLPHSON ${ }^{1}$ AND STEVEN SPERBER
}

\begin{abstract}
Consider the complex of differential forms on an open affine subvariety $U$ of $\mathbf{A}^{N}$ with differential $\omega \mapsto d \omega+\phi \wedge \omega$, where $d$ is the usual exterior derivative and $\phi$ is a fixed 1-form on $U$. For certain $U$ and $\phi$, we compute the cohomology of this complex.
\end{abstract}

\section{$\S 1$. Introduction}

For many purposes, a hypergeometric function (in any number of variables) may be thought of as an integral

$$
\int \frac{\exp g(x)}{f_{1}(x)^{\beta_{1}} \cdots f_{r}(x)^{\beta_{r}}} d x_{1} \wedge \cdots \wedge d x_{N}
$$

where $\beta_{1}, \ldots, \beta_{r} \in \mathbf{C}, g, f_{1}, \ldots, f_{r}$ are polynomials in $x_{1}, \ldots, x_{N}$, and integration is taken over some cycle. (The variables of the hypergeometric function occur as coefficients of the polynomials in the integrand.) This leads one to consider twisted de Rham cohomology: Take the complex of global differential forms on the complement of the divisor $f_{1} \cdots f_{r}=0$ and "twist" the usual exterior derivative $d$ by $f_{1}^{-\beta_{1}} \cdots f_{r}^{-\beta_{r}} \exp g$, i.e., replace $d$ by $d+\left(d g-\sum_{j=1}^{r} \beta_{j} d f_{j} / f_{j}\right) \wedge$. In this article, we compute the cohomology of this complex for generic $g, f_{1}, \ldots, f_{r}, \beta_{1}, \ldots, \beta_{r}$.

Recent work on this problem has been done by Kita $[\mathrm{KI}]$ and AomotoKita-Orlik-Terao [AKOT], to which we refer for further background and applications along the above lines. We take a somewhat different approach here. In [DW1], Dwork introduced a $p$-adic cohomology theory for varieties over finite fields, which is also often referred to as "twisted de Rham cohomology." Dwork's definition is algebraic and makes sense over any field of characteristic zero. The connection between Dwork's theory and classical de Rham cohomology was studied by Katz [K1, K2], who introduced an algebraic notion of "Laplace transform" to connect the two theories. This theory of the Laplace transform was developed further by Dwork [DW2 chapters 10 and 11] (see also Batyrev [B section 7]).

Received May 15, 1995.

${ }^{1}$ Partially supported by NSF grant no. DMS-9305514. 
Dwork's p-adic cohomology theory was developed further in [AS3, AS4], where the cohomology of a general class of "twisted exponential sums" was computed, and in [AS5], where the cohomology of smooth complete intersections over finite fields was computed. The point of this article is that, via the Laplace transform, the results and methods of [AS3, AS4, AS5] can be used to compute the twisted de Rham cohomology groups as defined in [KI, AKOT]. The work of Dwork establishes a connection between special values of ( $p$-adic) hypergeometric functions and eigenvalues of Frobenius acting on $p$-adic cohomology. We hope that our work here on the relation between Dwork cohomology and classical hypergeometric functions will ultimately yield new insights into this phenomenon.

We outline the method here. Introduce dummy variables $x_{N+1}, \ldots$, $x_{N+r}$ and consider the formal integral

$$
\int x_{N+1}^{\beta_{1}} \cdots x_{N+r}^{\beta_{r}} \exp \left(g+\sum_{j=1}^{r} x_{N+j} f_{j}\right) d x_{1} \wedge \cdots \wedge d x_{N+r} .
$$

Making the change of variable $x_{N+j} \mapsto x_{N+j} / f_{j}$ and integrating formally with respect to $x_{N+1}, \ldots, x_{N+r}$, we see that this is equal (up to $\Gamma$-factors) to (1.1). (This is referred to as the "Cayley trick" in [GKZ section 2.5].) This leads one to consider the complex of global differential forms in $x_{1}, \ldots, x_{N+r}$ on the complement of the divisor $x_{N+1} \cdots x_{N+r}=0$ with differential $d+$ $\left(d h+\sum_{j=1}^{r} \beta_{j} d x_{N+j} / x_{N+j}\right) \wedge$, where

$$
h=g+\sum_{j=1}^{r} x_{N+j} f_{j}
$$

This reduces us to the situation where poles occur along coordinate hyperplanes only.

The integral (1.2) is a formal analogue of a "twisted exponential sum" $\sum_{x}\left(\prod_{i} \chi_{i}\left(x_{i}\right)\right) \Psi(h(x))$, where $h$ is a polynomial over a finite field, $\chi_{i}$ (resp. $\Psi$ ) is a multiplicative (resp. additive) character of that finite field, and the sum runs over elements of the field. The $p$-adic cohomology of such sums was studied in [AS3, AS4]. The main point, which was the basis for those articles, is that cohomology can be computed from a much smaller complex (the complex $\hat{K}^{*}$ introduced in section 4 ), where questions about cohomology can often be answered by applying results of Kouchnirenko $[\mathrm{KO}]$.

We state our main result. The most natural setting is the purely toric case, although we ultimately give results for the "mixed case" (a product 
of toric and affine spaces) as well (Theorem 6.7). The toric case seems to cover all the classical hypergeometric functions (see [DL], particularly the appendix). For example, Appel's hypergeometric function $F_{4}$, which required some extra work from the point of view of $[\mathrm{KI}]$, fits nicely into this situation (see the example at the end of this section). Let $\mathbf{T}^{N}$ be the $N$-torus over a field $F$ of characteristic zero and let $g, f_{1}, \ldots, f_{r} \in$ $F\left[x_{1}, \ldots, x_{N},\left(x_{1} \cdots x_{N}\right)^{-1}\right]$. For any $f \in F\left[x_{1}, \ldots, x_{N},\left(x_{1} \cdots x_{N}\right)^{-1}\right]$, we define the support of $f, \operatorname{supp}(f)$, to be the set of exponents of the monomials appearing in $f$, thought of as lattice points in $\mathbf{R}^{N}$. Let $\Delta(h) \subseteq \mathbf{R}^{N+r}$ be the convex hull of $\operatorname{supp}(h) \cup\{(0, \ldots, 0)\}$, where $h$ is defined by (1.3). Let $Y \subseteq \mathbf{T}^{N}$ be the divisor $f_{1} \cdots f_{r}=0$ and let $\Omega^{l}(* Y)$ be the space of global $l$-forms with poles along $Y$. Let

$$
\nabla_{g, \alpha}=d+\left(d g+\sum_{i=1}^{N} \alpha_{i} \frac{d x_{i}}{x_{i}}-\sum_{j=1}^{r} \alpha_{N+j} \frac{d f_{j}}{f_{j}}\right) \wedge: \Omega^{l}(* Y) \longrightarrow \Omega^{l+1}(* Y)
$$

where $\alpha_{1}, \ldots, \alpha_{N+r} \in F$. This defines a complex $\left(\Omega^{\cdot}(* Y), \nabla_{g, \alpha}\right)$.

THEOREM 1.4. Suppose that $\alpha_{N+1}, \ldots, \alpha_{N+r} \notin \mathbf{Z}, h$ is nondegenerate relative to $\Delta(h)$, and $\operatorname{dim} \Delta(h)=N+r$. Then

$$
\begin{array}{rlrl}
H^{l}\left(\Omega^{*}(* Y), \nabla_{g, \alpha}\right) & =0 & (l \neq N), \\
\operatorname{dim}_{F} H^{N}\left(\Omega^{*}(* Y), \nabla_{g, \alpha}\right) & =(N+r) ! V(h),
\end{array}
$$

where $V(h)$ denotes the volume of $\Delta(h)$ relative to Lebesgue measure on $\mathbf{R}^{N+r}$.

Remark. The definition of " $h$ nondegenerate relative to $\Delta(h)$ " will be recalled in section 4 . It ensures that we are at an ordinary point of the corresponding system of hypergeometric differential equations ([A Lemma 3.3]). For now, we observe that (for $\left.\operatorname{specified} \operatorname{supp}(g), \operatorname{supp}\left(f_{j}\right), j=1, \ldots, r\right)$ this condition is satisfied for generic $g, f_{1}, \ldots, f_{r}$ ([KO Théorème 6.1]).

EXAMPLE. (see [KI section 5.4]) Appel's hypergeometric function $F_{4}$ has an integral representation of the form

$$
\int x_{1}^{\alpha_{1}} x_{2}^{\alpha_{2}}\left(1-x_{1}-x_{2}\right)^{\alpha_{3}}\left(1-\frac{\lambda_{1}}{x_{1}}-\frac{\lambda_{2}}{x_{2}}\right)^{\alpha_{4}} d x_{1} \wedge d x_{2},
$$

so we take $g=0, f_{1}=1-x_{1}-x_{2}, f_{2}=1-\lambda_{1} / x_{1}-\lambda_{2} / x_{2}$. The polytope $\Delta(h) \subseteq \mathbf{R}^{4}$ is the convex hull of the origin and $\operatorname{supp}\left(x_{3} f_{1}+x_{4} f_{2}\right)$. One 
computes $4 ! V(h)=4$. For generic $\lambda_{1}, \lambda_{2}, x_{3} f_{1}+x_{4} f_{2}$ is nondegenerate, hence for $\alpha_{3}, \alpha_{4} \notin \mathbf{Z}$ we have in that case

$$
\begin{aligned}
H^{l}\left(\Omega^{*}(* Y), \nabla_{\alpha}\right) & =0 \quad(l \neq 2), \\
\operatorname{dim}_{F} H^{2}\left(\Omega^{*}(* Y), \nabla_{\alpha}\right) & =4 .
\end{aligned}
$$

The latter equality reflects the fact that the system of partial differential equations satisfied by $F_{4}$ has four linearly independent solutions at an ordinary point.

Another example is given in section 7 , where we calculate the twisted de Rham cohomology on $\mathbf{A}^{N}$ for "generic" polynomials $g, f_{1}, \ldots, f_{r}$ of degrees $d_{0}, d_{1}, \ldots, d_{r}$, respectively. The special case $d_{i}=1$ for $i=1, \ldots, r$ was worked out in [AKOT].

\section{§2. Twisted de Rham complexes}

Let $F$ be a field of characteristic 0 , let $\mathbf{T}^{m}$ be the $m$-torus over $F$, and let $\mathbf{A}^{n}$ be affine $n$-space over $F$. Put $N=m+n$. Let $f_{1}, \ldots, f_{r}$, $g \in F\left[x_{1}, \ldots, x_{N},\left(x_{1} \cdots x_{m}\right)^{-1}\right]$, the coordinate ring of $\mathbf{T}^{m} \times \mathbf{A}^{n}$, and let $Y \subseteq \mathbf{T}^{m} \times \mathbf{A}^{n}$ be the divisor $f_{1} \cdots f_{r}=0$. (We allow the possibilities $m=0$ and $n=0$.) Let $\Omega^{l}(* Y)$ be the space of global $l$-forms with poles along $Y$. Thus

$$
\Omega^{0}(* Y)=F\left[x_{1}, \ldots, x_{N},\left(x_{1} \cdots x_{m} f_{1} \cdots f_{r}\right)^{-1}\right]
$$

and $\Omega^{l}(* Y)$ is the free $\Omega^{0}(* Y)$-module with basis

$$
\frac{d x_{i_{1}}}{x_{i_{1}}} \wedge \cdots \wedge \frac{d x_{i_{k}}}{x_{i_{k}}} \wedge d x_{i_{k+1}} \wedge \cdots \wedge d x_{i_{l}},
$$

where $1 \leq i_{1}<\cdots<i_{k} \leq m$, and $m+1 \leq i_{k+1}<\cdots<i_{l} \leq m+n$. Choose $\alpha=\left(\alpha_{1}, \ldots, \alpha_{N+r}\right) \in F^{N+r}$ subject to the requirement that $\alpha_{m+1}=\cdots=$ $\alpha_{m+n}=0$. Let $\omega_{g, \alpha} \in \Omega^{1}(* Y)$ be given by

$$
\omega_{g, \alpha}=d g+\sum_{i=1}^{N} \alpha_{i} \frac{d x_{i}}{x_{i}}-\sum_{j=1}^{r} \alpha_{N+j} \frac{d f_{j}}{f_{j}}
$$

where $d: \Omega^{l}(* Y) \rightarrow \Omega^{l+1}(* Y)$ is the usual exterior derivative, and put

$$
\nabla_{g, \alpha}=d+\omega_{g, \alpha} \wedge: \Omega^{l}(* Y) \longrightarrow \Omega^{l+1}(* Y) .
$$

Straightforward calculations show that $\left(\Omega^{\cdot}(* Y), \nabla_{g, \alpha}\right)$ is a complex. 
We shall compute the cohomology of this complex for sufficiently general $f_{1}, \ldots, f_{r}, g$ and nonintegral $\alpha_{N+1}, \ldots, \alpha_{N+r}$. The first step is to apply the Laplace transform theory of Dwork and Katz. Introduce dummy variables $x_{N+1}, \ldots, x_{N+r}$ and put $R=F\left[x_{1}, \ldots, x_{N+r},\left(x_{1} \cdots x_{m}\right)^{-1}\right]$, the coordinate ring of $\mathbf{T}^{m} \times \mathbf{A}^{n+r}$. Put

$$
\begin{aligned}
h\left(x_{1}, \ldots, x_{N+r}\right)=g\left(x_{1}, \ldots, x_{N}\right)+ & x_{N+1} f_{1}\left(x_{1}, \ldots, x_{N}\right) \\
& +\cdots+x_{N+r} f_{r}\left(x_{1}, \ldots, x_{N}\right) \in R .
\end{aligned}
$$

We need some notation to distinguish the roles played by the different types of variables that are involved. We index the set of all variables by $S$ $\{1, \ldots, N+r\}$, the toric variables by $S_{\text {to }}=\{1, \ldots, m\}$, the affine variables by $S_{\mathrm{af}}=\{m+1, \ldots, N+r\}$, the space variables by $S_{\mathrm{sp}}=\{1, \ldots, N\}$, and the dummy variables by $S_{\mathrm{du}}=\{N+1, \ldots, N+r\}$. For any subset $I \subseteq S$, we use subscripts to denote intersection with one of these sets, e.g., $I_{\text {to }}=I \cap S_{\text {to }}$. We also put $I_{\mathrm{sp}}^{\text {af }}=I \cap S_{\mathrm{af}} \cap S_{\mathrm{sp}}$. For any subset $I \subseteq S_{\mathrm{af}}$, let $R^{I}=\left(\prod_{i \in I} x_{i}\right) R$, the set of elements of $R$ divisible by $x_{i}$ for all $i \in I$.

We introduce the ring $R^{\prime}=R\left[\left(\prod_{j=1}^{r} x_{N+j}\right)^{-1}\right]$ and put $R^{\prime I}=\left(\prod_{i \in I} x_{i}\right) R^{\prime}$ for any subset $I \subseteq S_{\mathrm{sp}} \cap S_{\text {af }}$. Let $Z \subseteq \mathbf{T}^{m} \times \mathbf{A}^{n+r}$ be the divisor $\prod_{j=1}^{r} x_{N+j}=$ 0 and let $\Omega^{l}(* Z)$ be the space of global $l$-forms with poles along $Z$. Thus $\Omega^{0}(* Z)=R^{\prime}$ and $\Omega^{l}(* Z)$ is the free $R^{\prime}$-module with basis

$$
\left(\prod_{i_{j} \in S_{\mathrm{af}} \cap S_{\mathrm{sp}}} x_{i_{\jmath}}\right) \frac{d x_{i_{1}}}{x_{i_{1}}} \wedge \cdots \wedge \frac{d x_{i_{l}}}{x_{i_{l}}}, \quad 1 \leq i_{1}<\cdots<i_{l} \leq N+r
$$

i.e.,

$$
\Omega^{l}(* Z)=\underset{1 \leq i_{1}<\cdots<i_{l} \leq N+r}{ } R^{\prime I_{\mathrm{sp}}^{\mathrm{af}}} \frac{d x_{i_{1}}}{x_{i_{1}}} \wedge \cdots \wedge \frac{d x_{i_{l}}}{x_{i_{l}}}
$$

where $I=\left\{i_{1}, \ldots, i_{l}\right\}$. We define the differential to be

$$
\delta_{h, \alpha}=d+\left(d h+\sum_{i=1}^{N+r} \alpha_{i} \frac{d x_{i}}{x_{i}}\right) \wedge .
$$

Straightforward calculations show that $\left(\Omega^{*}(* Z), \delta_{h, \alpha}\right)$ is a complex.

We give an explicit formula for $\delta_{h, \alpha}$. For $i=1, \ldots, N+r$, define differential operators $D_{i, h, \alpha}$ by

$$
D_{i, h, \alpha}=x_{i} \frac{\partial}{\partial x_{i}}+\alpha_{i}+x_{i} \frac{\partial h}{\partial x_{i}} .
$$


Note that for $i=1, \ldots, N$,

$$
x_{i} \frac{\partial h}{\partial x_{i}}=x_{i} \frac{\partial g}{\partial x_{i}}+\sum_{j=1}^{r} x_{N+j} x_{i} \frac{\partial f_{j}}{\partial x_{i}}
$$

while for $j=1, \ldots, r$,

$$
x_{N+j} \frac{\partial h}{\partial x_{N+j}}=x_{N+j} f_{j} .
$$

For $\xi\left(d x_{i_{1}} / x_{i_{1}}\right) \wedge \cdots \wedge\left(d x_{i_{l}} / x_{i_{l}}\right) \in \Omega^{l}(* Z)$ we have

$$
\delta_{h, \alpha}\left(\xi \frac{d x_{i_{1}}}{x_{i_{1}}} \wedge \cdots \wedge \frac{d x_{i_{l}}}{x_{i_{l}}}\right)=\left(\sum_{i=1}^{N+r} D_{i, h, \alpha}(\xi) \frac{d x_{i}}{x_{i}}\right) \wedge \frac{d x_{i_{1}}}{x_{i_{1}}} \wedge \cdots \wedge \frac{d x_{i_{l}}}{x_{i_{l}}}
$$

We define a "direct image complex" $\tilde{\Omega} \cdot(* Z)$ under the projection of $\mathbf{T}^{m} \times \mathbf{A}^{n+r}$ onto the first $N$ factors as follows. For $l=0, \ldots, N$, put

$$
\tilde{\Omega}^{l}(* Z)=\bigoplus_{1 \leq i_{1}<\cdots<i_{l} \leq N}\left(R^{\prime I_{\mathrm{af}}} / \sum_{j=1}^{r} D_{N+j, h, \alpha}\left(R^{\prime I_{\mathrm{af}}}\right)\right) \frac{d x_{i_{1}}}{x_{i_{1}}} \wedge \cdots \wedge \frac{d x_{i_{l}}}{x_{i_{l}}},
$$

where $I=\left\{i_{1}, \ldots, i_{l}\right\}$. Define $\tilde{\delta}_{h, \alpha}: \tilde{\Omega}^{l}(* Z) \rightarrow \tilde{\Omega}^{l+1}(* Z)$ by additivity and the formula

$$
\tilde{\delta}_{h, \alpha}\left(\xi \frac{d x_{i_{1}}}{x_{i_{1}}} \wedge \cdots \wedge \frac{d x_{i_{l}}}{x_{i_{l}}}\right)=\left(\sum_{i=1}^{N} D_{i, h, \alpha}(\xi) \frac{d x_{i}}{x_{i}}\right) \wedge \frac{d x_{i_{1}}}{x_{i_{1}}} \wedge \cdots \wedge \frac{d x_{i_{l}}}{x_{i_{l}}}
$$

a well-defined map since all the $D_{i, h, \alpha}$ 's commute with one another.

We shall show that the complex $\left(\tilde{\Omega}^{\cdot}(* Z), \tilde{\delta}_{h, \alpha}\right)$ is isomorphic to the complex $\left(\Omega^{\cdot}(* Y), \nabla_{g, \alpha}\right)$ when $\alpha_{N+1}, \ldots, \alpha_{N+r}$ are not integers. Let $L$ : $R^{\prime} \rightarrow \Omega^{0}(* Y)$ be defined by $F$-linearity and the condition

$$
\begin{aligned}
& L\left(x^{u} x_{N+1}^{u_{N+1}} \cdots x_{N+r}^{u_{N+r}}\right) \\
& \quad=(-1)^{\sum_{j=1}^{r} u_{N+j}}\left(\prod_{j=1}^{r}\left(\alpha_{N+j}+1\right)_{u_{N+j}-1}\right) x^{u} f_{1}^{-u_{N+1}} \cdots f_{r}^{-u_{N+r}},
\end{aligned}
$$

where $u=\left(u_{1}, \ldots, u_{N}\right),\left(u_{1}, \ldots, u_{N+r}\right) \in \mathbf{Z}^{N+r}, u_{m+1}, \ldots, u_{N} \geq 0$, and

$$
\begin{aligned}
& \left(\alpha_{N+j}+1\right)_{u_{N+j}-1} \\
& \quad= \begin{cases}\left(\alpha_{N+j}+1\right)\left(\alpha_{N+j}+2\right) \cdots\left(\alpha_{N+j}+u_{N+j}-1\right) & \text { if } u_{N+j}>1 \\
1 & \text { if } u_{N+j}=1 \\
\left(\alpha_{N+j}\left(\alpha_{N+j}-1\right) \cdots\left(\alpha_{N+j}+u_{N+j}\right)\right)^{-1} & \text { if } u_{N+j}<1\end{cases}
\end{aligned}
$$

a well-defined element of $F$ since $\alpha_{N+j} \notin \mathbf{Z}$. 
LEMmA 2.5. Suppose $\alpha_{N+1}, \ldots, \alpha_{N+r}$ are not integers. Then the map $L$ induces an isomorphism $\bar{L}: R^{\prime} / \sum_{j=1}^{r} D_{N+j, h, \alpha}\left(R^{\prime}\right) \simeq \Omega^{0}(* Y)$.

Proof (Dwork). For $i=0,1, \ldots, r$, set

$$
R^{(i)}=F\left[x_{1}, \ldots, x_{N+i},\left(x_{1} \cdots x_{m} x_{N+1} \cdots x_{N+i} f_{i+1} \cdots f_{r}\right)^{-1}\right] .
$$

In particular, $R^{(r)}=R^{\prime}$ and $R^{(0)}=\Omega^{0}(* Y)$. Note that $D_{N+j, h, \alpha}$ acts on $R^{(i)}$ for $j=1, \ldots, i$. We show that for $i=1, \ldots, r$ there are isomorphisms of $F$-vector spaces

$$
R^{(i)} / D_{N+i, h, \alpha}\left(R^{(i)}\right) \simeq R^{(i-1)}
$$

It will be clear that under this isomorphism the action of $D_{N+j, h, \alpha}$ on $R^{(i)} / D_{N+i, h, \alpha}\left(R^{(i)}\right)$ is identified with its action on $R^{(i-1)}$ for $j=1, \ldots, i-1$. We then get the desired isomorphism by composition.

Note that $R^{(i)}$ is a free module over the ring $F\left[x_{1}, \ldots, x_{N+i-1},\left(x_{1} \ldots\right.\right.$ $\left.\left.x_{m} x_{N+1} \cdots x_{N+i-1} f_{i+1} \cdots f_{r}\right)^{-1}\right]$ with basis $\left\{x_{N+i}^{u} \mid u \in \mathbf{Z}\right\}$ and $R^{(i-1)}$ is a module over this ring spanned by $\left\{f_{i}^{-u}\right\}_{u=0}^{\infty}$. For $i=1, \ldots, r$, we define $L_{i}: R^{(i)} \rightarrow R^{(i-1)}$ to be the homomorphism of modules over this ring defined by

$$
L_{i}\left(x_{N+i}^{u}\right)=(-1)^{u}\left(\alpha_{N+i}+1\right)_{u-1} f_{i}^{-u}
$$

for $u \in \mathbf{Z}$. Each $L_{i}$ is $F$-linear and surjective (since $\alpha_{N+i} \notin \mathbf{Z}$ ) and it is easily checked that $\bar{L}=L_{1} \circ \cdots \circ L_{r}$. We have

$$
\begin{aligned}
& D_{N+i, h, \alpha}\left(\frac{x^{u} x_{N+1}^{u_{N+1}} \cdots x_{N+i}^{u_{N+\imath}}}{f_{i+1}^{u_{N+i+1}} \cdots f_{r}^{u_{N+r}}}\right) \\
& \quad=\left(u_{N+i}+\alpha_{N+i}\right) \frac{x^{u} x_{N+1}^{u_{N+1}} \cdots x_{N+i}^{u_{N+i}}}{f_{i+1}^{u_{N+1}} \cdots f_{r}^{u_{N+r}}}+\frac{f_{i} x^{u} x_{N+1}^{u_{N+1}} \cdots x_{N+i-1}^{u_{N+i-1}} x_{N+i}^{u_{N+1}}}{f_{i+1}^{u_{N+1+1}} \cdots f_{r}^{u_{N+r}}}
\end{aligned}
$$

and an easy calculation shows that $D_{N+i, h, \alpha}\left(R^{(i)}\right) \subseteq \operatorname{ker} L_{i}$.

We show that $\operatorname{ker} L_{i} \subseteq D_{N+i, h, \alpha}\left(R^{(i)}\right)$. Let $\xi \in \operatorname{ker} L_{i}$ and write

$$
\begin{aligned}
\xi & =\sum_{u} c_{u} \frac{x^{u} x_{N+1}^{u_{N+1}} \cdots x_{N+i}^{u_{N+i}}}{f_{i+1}^{u_{N+1+1}} \cdots f_{r}^{u_{N+r}}} \quad\left(c_{u} \in F\right) \\
& =\sum_{k=M_{1}}^{M_{2}} x_{N+i}^{k} q_{k},
\end{aligned}
$$


where $M_{1}, M_{2} \in \mathbf{Z}$ and $q_{k} \in F\left[x_{1}, \ldots, x_{N+i-1}, \quad\left(x_{1} \cdots x_{m} x_{N+1} \cdots\right.\right.$ $\left.\left.x_{N+i-1} f_{i+1} \cdots f_{r}\right)^{-1}\right]$. We argue by induction on $M_{2}-M_{1}$. Write $\xi=$ $x_{N+i}^{M_{2}} q_{M_{2}}+\xi_{1}$, where $\xi_{1}=\sum_{k=M_{1}}^{M_{2}-1} x_{N+1}^{k} q_{k}$. We have

$$
\begin{aligned}
0 & =L_{i}(\xi) \\
& =\sum_{k=M_{1}}^{M_{2}}(-1)^{k}\left(\alpha_{N+i}+1\right)_{k-1} \frac{q_{k}}{f_{i}^{k}} .
\end{aligned}
$$

Solving this equation for $q_{M_{2}}$ (which is possible since $\alpha_{N+i}$ is not an integer) we get $q_{M_{2}}=f_{i} \xi_{2}$, where $\xi_{2} \in F\left[x_{1}, \ldots, x_{N+i-1},\left(x_{1} \cdots x_{m} x_{N+1} \cdots\right.\right.$ $\left.x_{N+i-1} f_{i+1} \cdots f_{r}\right)^{-1}$. Thus $\xi=\xi_{1}+x_{N+i}^{M_{2}} f_{i} \xi_{2}$, with $\xi_{2}$ independent of $x_{N+i}$. But

$$
D_{N+i, h, \alpha}\left(x_{N+i}^{M_{2}-1} \xi_{2}\right)=\left(\alpha_{N+i}+M_{2}-1\right) x_{N+i}^{M_{2}-1} \xi_{2}+x_{N+i}^{M_{2}} f_{i} \xi_{2}
$$

so

$$
\xi=\xi_{1}-\left(\alpha_{N+i}+M_{2}-1\right) x_{N+i}^{M_{2}-1} \xi_{2}+D_{N+i, h, \alpha}\left(x_{N+i}^{M_{2}-1} \xi_{2}\right) .
$$

Applying $L_{i}$ gives

$$
L_{i}\left(\xi_{1}-\left(\alpha_{N+i}+M_{2}-1\right) x_{N+i}^{M_{2}-1} \xi_{2}\right)=0 .
$$

We have

$$
\xi_{1}-\left(\alpha_{N+i}+M_{2}-1\right) x_{N+i}^{M_{2}-1} \xi_{2}=\sum_{k=M_{1}}^{M_{2}-1} x_{N+i}^{k} \tilde{q}_{k}
$$

with $\tilde{q}_{k} \in F\left[x_{1}, \ldots, x_{N+i-1},\left(x_{1} \cdots x_{m} x_{N+1} \cdots x_{N+i-1} f_{i+1} \cdots f_{r}\right)^{-1}\right]$. By induction we are reduced to the case $M_{1}=M_{2}=M$, i.e., $\xi=x_{N+i}^{M} q_{M}$. But from (2.6), we see that this implies $q_{M}=0$.

We regard $\bar{L}$ as an isomorphism between $\tilde{\Omega}^{0}(* Z)$ and $\Omega^{0}(* Y)$. We now explain how to use $\bar{L}$ to construct isomorphisms (also denoted $\bar{L}$ ) between $\tilde{\Omega}^{l}(* Z)$ and $\Omega^{l}(* Y)$ for $l=1, \ldots, N$ as well. Since multiplications by $x_{1}, \ldots, x_{N}$ commute with $D_{N+1, h, \alpha}, \ldots, D_{N+r, h, \alpha}$, we have isomorphisms

$$
R^{\prime} / \sum_{j=1}^{r} D_{N+j, h, \alpha}\left(R^{\prime}\right) \simeq R^{\prime I_{\mathrm{af}}} / \sum_{j=1}^{r} D_{N+j, h, \alpha}\left(R^{\prime I_{\mathrm{af}}}\right)
$$

given by multiplication by $\prod_{i \in I_{\mathrm{af}}} x_{i}$, where $I=\left\{i_{1}, \ldots, i_{l}\right\} \subseteq S_{\mathrm{sp}}$. Thus $\tilde{\Omega}^{l}(* Z)$ can be identified with

$$
\bigoplus_{1 \leq i_{1}<\cdots<i_{l} \leq N}\left(R^{\prime} / \sum_{j=1}^{r} D_{N+j, h, \alpha}\left(R^{\prime}\right)\right) \frac{d x_{i_{1}}}{x_{i_{1}}} \wedge \cdots \wedge \frac{d x_{i_{k}}}{x_{i_{k}}} \wedge d x_{i_{k+1}} \wedge \cdots \wedge d x_{i_{l}}
$$


where $1 \leq i_{1}<\cdots<i_{k} \leq m, m+1 \leq i_{k+1}<\cdots<i_{l} \leq N$. For $\xi \in R^{\prime} / \sum_{j=1}^{r} D_{N+j, h, \alpha}\left(R^{\prime}\right)$, define

$$
\begin{aligned}
\bar{L}\left(\xi \frac{d x_{i_{1}}}{x_{i_{1}}} \wedge \cdots \wedge \frac{d x_{i_{k}}}{x_{i_{k}}} \wedge d x_{i_{k+1}}\right. & \left.\wedge \cdots \wedge d x_{i_{l}}\right) \\
& =\bar{L}(\xi) \frac{d x_{i_{1}}}{x_{i_{1}}} \wedge \cdots \wedge \frac{d x_{i_{k}}}{x_{i_{k}}} \wedge d x_{i_{k+1}} \wedge \cdots \wedge d x_{i_{l}}
\end{aligned}
$$

By Lemma 2.5 and the fact that $\Omega^{l}(* Y)$ is a free $\Omega^{0}(* Y)$-module with basis given by $(2.1)$, it follows that $\bar{L}: \tilde{\Omega}^{l}(* Z) \rightarrow \Omega^{l}(* Y)$ is an isomorphism of $F$-vector spaces for $l=0,1, \ldots, N$ (provided $\alpha_{N+1}, \ldots, \alpha_{N+r}$ are not integers).

THEOREM 2.8. Suppose $\alpha_{N+1}, \ldots, \alpha_{N+r}$ are not integers. Then the map $\bar{L}: \tilde{\Omega}^{\cdot}(* Z) \rightarrow \Omega^{*}(* Y)$ is an isomorphism of complexes.

Proof. By what we have done so far, it suffices to show that $\bar{L}$ is a map of complexes, i.e., that the diagram

$$
\begin{array}{ccc}
\tilde{\Omega}^{l}(* Z) & \stackrel{\tilde{\delta}_{h, \alpha}}{\longrightarrow} & \tilde{\Omega}^{l+1}(* Z) \\
\bar{L} \downarrow & & \downarrow \bar{L} \\
\Omega^{l}(* Y) & \stackrel{\nabla_{g, \alpha}}{\longrightarrow} & \Omega^{l+1}(* Y)
\end{array}
$$

commutes. Let $\xi=x^{u} x_{N+1}^{u_{N+1}} \cdots x_{N+r}^{u_{N+r}} \in R^{\prime}$ and let $[\xi]$ denote its image in $R^{\prime} / \sum_{j=1}^{r} D_{N+j, h, \alpha}\left(R^{\prime}\right)$. By $(2.7)$, elements of $\tilde{\Omega}^{l}(* Z)$ may be represented as linear combinations of expressions of the form

$$
[\xi] \frac{d x_{i_{1}}}{x_{i_{1}}} \wedge \cdots \wedge \frac{d x_{i_{k}}}{x_{i_{k}}} \wedge d x_{i_{k+1}} \wedge \cdots \wedge d x_{i_{l}} .
$$

We need to check that

$$
\begin{aligned}
\bar{L} \circ \tilde{\delta}_{h, \alpha}([\xi] & \left.\frac{d x_{i_{1}}}{x_{i_{1}}} \wedge \cdots \wedge \frac{d x_{i_{k}}}{x_{i_{k}}} \wedge d x_{i_{k+1}} \wedge \cdots \wedge d x_{i_{l}}\right) \\
& =\nabla_{g, \alpha} \circ \bar{L}\left([\xi] \frac{d x_{i_{1}}}{x_{i_{1}}} \wedge \cdots \wedge \frac{d x_{i_{k}}}{x_{i_{k}}} \wedge d x_{i_{k+1}} \wedge \cdots \wedge d x_{i_{l}}\right) .
\end{aligned}
$$

Let $s \in S_{\mathrm{sp}}$. We compute the coefficient of

$$
d x_{s} \wedge \frac{d x_{i_{1}}}{x_{i_{1}}} \wedge \cdots \wedge \frac{d x_{i_{k}}}{x_{i_{k}}} \wedge d x_{i_{k+1}} \wedge \cdots \wedge d x_{i_{l}}
$$


on both sides of (2.9). First of all, the exterior product (2.10) vanishes if $s \in I=\left\{i_{1}, \ldots, i_{l}\right\}$, so we may assume $s \notin I$. By definition, the coefficient of $\frac{d x_{s}}{x_{s}} \wedge \frac{d x_{i_{1}}}{x_{i_{1}}} \wedge \cdots \wedge \frac{d x_{i_{k}}}{x_{i_{k}}} \wedge d x_{i_{k+1}} \wedge \cdots \wedge d x_{i_{l}}$ in $\tilde{\delta}_{h, \alpha}\left([\xi] \frac{d x_{i_{1}}}{x_{i_{1}}} \wedge \cdots \wedge \frac{d x_{i_{k}}}{x_{i_{k}}} \wedge\right.$ $\left.d x_{i_{k+1}} \wedge \cdots \wedge d x_{i_{l}}\right)$ is $\left[D_{s, h, \alpha}\left(x^{u} x_{N+1}^{u_{N+1}} \cdots x_{N+r}^{u_{N+r}}\right)\right]$, and

$$
\begin{aligned}
D_{s, h, \alpha}\left(x^{u} x_{N+1}^{u_{N+1}}\right. & \left.\cdots x_{N+r}^{u_{N+r}}\right) \\
= & \left(u_{s}+\alpha_{s}+x_{s} \frac{\partial g}{\partial x_{s}}\right) x^{u} x_{N+1}^{u_{N+1}} \cdots x_{N+r}^{u_{N+r}} \\
& +\sum_{j=1}^{r} x_{N+j} x_{s} \frac{\partial f_{j}}{\partial x_{s}} x^{u} x_{N+1}^{u_{N+1}} \cdots x_{N+r}^{u_{N+r}} .
\end{aligned}
$$

Thus the coefficient of (2.10) on the left-hand side of $(2.9)$ is

$$
\begin{aligned}
& \frac{\left(u_{s}+\alpha_{s}+x_{s} \partial g / \partial x_{s}\right)(-1)^{\sum_{\jmath=1}^{r} u_{N+\jmath}}\left(\prod_{j=1}^{r}\left(\alpha_{N+j}+1\right)_{u_{N+j}-1}\right) x^{u}}{x_{s} f_{1}^{u_{N+1}} \cdots f_{r}^{u_{N+r}}} \\
& -\frac{(-1)^{\sum_{\jmath=1}^{r} u_{N+j}}\left(\prod_{j=1}^{r}\left(\alpha_{N+j}+1\right)_{u_{N+j}-1}\right) x^{u}}{f_{1}^{u_{N+1}} \cdots f_{r}^{u_{N+r}}} \sum_{j=1}^{r}\left(\alpha_{N+j}+u_{N+j}\right) \frac{\partial f_{j} / \partial x_{s}}{f_{j}} .
\end{aligned}
$$

Note that if $s \in S_{\mathrm{af}}$, then $\alpha_{s}=0$ so $\left(u_{s}+\alpha_{s}\right) x^{u}$ is divisible by $x_{s}$. Now consider the right-hand side of (2.9). We have

$$
\begin{gathered}
\bar{L}\left([\xi] \frac{d x_{i_{1}}}{x_{i_{1}}} \wedge \cdots \wedge \frac{d x_{i_{k}}}{x_{i_{k}}} \wedge d x_{i_{k+1}} \wedge \cdots \wedge d x_{i_{l}}\right) \\
=\frac{(-1)^{\sum_{\jmath=1}^{r} u_{N+j}}\left(\prod_{j=1}^{r}\left(\alpha_{N+j}+1\right)_{u_{N+\jmath}-1}\right) x^{u}}{f_{1}^{u_{N+1}} \cdots f_{r}^{u_{N+r}}} \\
\cdot \frac{d x_{i_{1}}}{x_{i_{1}}} \wedge \cdots \wedge \frac{d x_{i_{k}}}{x_{i_{k}}} \wedge d x_{i_{k+1}} \wedge \cdots \wedge d x_{i_{l}} .
\end{gathered}
$$

Applying $\nabla_{g, \alpha}$ to the right-hand side of (2.12) and picking out the coefficient 
of (2.10) we get

$$
\begin{aligned}
&\left(\frac{\partial}{\partial x_{s}}+\frac{\partial g}{\partial x_{s}}+\frac{\alpha_{s}}{x_{s}}-\sum_{j=1}^{r}\right.\left.\alpha_{N+j} \frac{\partial f_{j} / \partial x_{s}}{f_{j}}\right) \\
& \cdot\left(\frac{(-1)^{\sum_{j=1}^{r} u_{N+j}}\left(\prod_{j=1}^{r}\left(\alpha_{N+j}+1\right)_{u_{N+3}-1}\right) x^{u}}{f_{1}^{u_{N+1}} \cdots f_{r}^{u_{N+r}}}\right) .
\end{aligned}
$$

A short calculation shows that this is equal to (2.11).

\section{$\S$ 3. Relation between $\tilde{\Omega}^{\cdot}(* Z)$ and $\Omega^{*}(* Z)$}

Our methods will compute the cohomology of $\Omega^{*}(* Z)$ (Theorem 5.1). In view of Theorem 2.8, we must thus establish a connection between the cohomology of $\tilde{\Omega}^{\cdot}(* Z)$ and the cohomology of $\Omega^{\cdot}(* Z)$. The hypothesis of the following theorem might be awkward to check directly, but we shall show in section 5 that it is a consequence of the hypothesis that allows us to compute the cohomology of $\Omega^{*}(* Z)$.

For $I \subseteq S_{\mathrm{sp}} \cap S_{\mathrm{af}}$, define a complex $\left(A_{I}^{*}, \delta\right)$ by

$$
\begin{gathered}
A_{I}^{l}=\bigoplus_{N+1 \leq j_{1}<\cdots<j_{l} \leq N+r} R^{\prime I} \frac{d x_{j_{1}}}{x_{j_{1}}} \wedge \cdots \wedge \frac{d x_{j_{l}}}{x_{j_{l}}}, \\
\delta\left(\xi \frac{d x_{j_{1}}}{x_{j_{1}}} \wedge \cdots \wedge \frac{d x_{j_{l}}}{x_{j_{l}}}\right)=\left(\sum_{k=N+1}^{N+r} D_{k, h, \alpha}(\xi) \frac{d x_{k}}{x_{k}}\right) \wedge \frac{d x_{j_{1}}}{x_{j_{1}}} \wedge \cdots \wedge \frac{d x_{j_{l}}}{x_{j_{l}}} .
\end{gathered}
$$

Note that for $j \in S_{\mathrm{af}} \cap S_{\mathrm{sp}}$, multiplication by $x_{j}$ commutes with $D_{k, h, \alpha}$ for $k=N+1, \ldots, N+r$, hence multiplication by $\prod_{j \in I} x_{j}$ is an isomorphism of complexes from $\left(A_{\emptyset}^{\cdot}, \delta\right)$ onto $\left(A_{I}^{*}, \delta\right)$. In particular, all these complexes have isomorphic cohomology.

Proposition 3.1. Suppose that $H^{l}\left(A_{\emptyset}\right)=0$ for $l \neq r$. Then $H^{l}(\tilde{\Omega} \cdot(* Z))=H^{l+r}(\Omega(* Z))$ for all $l$.

Remark. Up to reindexing and some sign changes, the complex $\left(A_{I}^{*}, \delta\right)$ is the Koszul complex on $R^{\prime I}$ defined by $\left\{D_{N+k, h, \alpha}\right\}_{k=1}^{r}$. Thus the hypothesis of the proposition is equivalent to the requirement that the Koszul complex $\left(A_{\emptyset}^{*}, \delta\right)$ be acyclic in positive dimension, i.e., $H_{l}=0$ for all $l>0$.

Proof. Consider the double complex ${ }^{K} p, q$ for $p, q \geq 0$ defined by

$$
\begin{aligned}
{ }^{K} p, q=\bigoplus_{1 \leq i_{1}<\cdots<i_{p} \leq N} \underset{N+1 \leq j_{1}<\cdots<j_{q} \leq N+r}{\bigoplus} R^{I_{\mathrm{af}}} \\
\quad \frac{d x_{i_{1}}}{x_{i_{1}}} \wedge \cdots \wedge \frac{d x_{i_{p}}}{x_{i_{p}}} \wedge \frac{d x_{j_{1}}}{x_{j_{1}}} \wedge \cdots \wedge \frac{d x_{j_{q}}}{x_{j_{q}}}
\end{aligned}
$$


where $I=\left\{i_{1}, \ldots, i_{p}\right\}$, with maps $d_{1}:{ }^{K} p, q \rightarrow{ }^{K} p+1, q, d_{2}:{ }^{K} p, q \rightarrow$ ${ }^{K} p, q+1$ defined by

$$
\begin{aligned}
& d_{1}\left(\xi \frac{d x_{i_{1}}}{x_{i_{1}}} \wedge \cdots \wedge \frac{d x_{i_{p}}}{x_{i_{p}}} \wedge \frac{d x_{j_{1}}}{x_{j_{1}}} \wedge \cdots \wedge \frac{d x_{j_{q}}}{x_{j_{q}}}\right) \\
& =\left(\sum_{k=1}^{N} D_{k, h, \alpha}(\xi) \frac{d x_{k}}{x_{k}}\right) \wedge \frac{d x_{i_{1}}}{x_{i_{1}}} \wedge \cdots \wedge \frac{d x_{i_{p}}}{x_{i_{p}}} \wedge \frac{d x_{j_{1}}}{x_{j_{1}}} \wedge \cdots \wedge \frac{d x_{j_{q}}}{x_{j_{q}}} \\
& d_{2}\left(\xi \frac{d x_{i_{1}}}{x_{i_{1}}} \wedge \cdots \wedge \frac{d x_{i_{p}}}{x_{i_{p}}} \wedge \frac{d x_{j_{1}}}{x_{j_{1}}} \wedge \cdots \wedge \frac{d x_{j_{q}}}{x_{j_{q}}}\right) \\
& =\left((-1)^{p} \sum_{k=N+1}^{N+r} D_{k, h, \alpha}(\xi) \frac{d x_{k}}{x_{k}}\right) \wedge \frac{d x_{i_{1}}}{x_{i_{1}}} \wedge \cdots \wedge \frac{d x_{i_{p}}}{x_{i_{p}}} \wedge \frac{d x_{j_{1}}}{x_{j_{1}}} \wedge \cdots \wedge \frac{d x_{j_{q}}}{x_{j_{q}}}
\end{aligned}
$$

The total complex associated to this double complex is easily seen to be $\Omega^{\cdot}(* Z)$. Let $X^{\cdot}$ be the complex $H^{r}\left({ }^{K} \cdot, \cdot d_{2}\right)$, i.e., $X^{p}={ }^{K} p, r / d_{2}\left({ }^{K} p, r-1\right)$ with differential induced by $d_{1}:{ }^{K} p, r \rightarrow{ }^{K} p+1, r$. It is easily seen that $\tilde{\Omega}^{\cdot}(* Z)=X^{*}$. Note that for a fixed $p$, the complex $\left({ }^{K} p, \cdot, d_{2}\right)$ satisfies

$$
\left({ }^{K} p, \cdot, d_{2}\right)=\bigoplus_{1 \leq i_{1}<\cdots<i_{p} \leq N}\left(A_{I_{\mathrm{af}}}^{\cdot},(-1)^{p} \delta\right)
$$

where $I=\left\{i_{1}, \ldots, i_{p}\right\}$. Our hypothesis implies that for all $I \subseteq S_{\mathrm{af}} \cap S_{\mathrm{sp}}$, $H^{l}\left(A_{I}^{*}\right)=0$ for $l \neq r$, hence $H^{q}\left({ }^{K} p, \cdot, d_{2}\right)=0$ for all $p$ and all $q \neq r$. The conclusion of the theorem is then a standard fact about double complexes [M Appendix B].

\section{$\S 4$. Cohomology of a related complex}

We consider a slightly more general version of the previous situation, but work in the purely toric case. This has the advantage that all the complexes we encounter are Koszul complexes (up to reindexing and sign changes), hence are somewhat easier to analyze. Let $f=\sum_{j \in J} a_{j} x^{j} \in$ $F\left[x_{1}, \ldots, x_{p},\left(x_{1} \cdots x_{p}\right)^{-1}\right]$, where $J \subseteq \mathbf{Z}^{p}$ is finite, $j=\left(j_{1}, \ldots, j_{p}\right), x^{j}=$ $x_{1}^{j_{1}} \cdots x_{p}^{j_{p}}$, and $a_{j} \in F^{\times}$. Let $\Delta(f) \subseteq \mathbf{R}^{p}$ be the convex hull of $J \cup$ $\{(0, \ldots, 0)\}$. Recall $[\mathrm{KO}]$ that $f$ is nondegenerate relative to $\Delta(f)$ if for every face $\sigma$ of $\Delta(f)$ not containing the origin, the Laurent polynomials $\partial f_{\sigma} / \partial x_{1}, \ldots, \partial f_{\sigma} / \partial x_{p}$ have no common zero in $\left(\bar{F}^{\times}\right)^{p}$, where $f_{\sigma}=$ $\sum_{j \in \sigma \cap J} a_{j} x^{j}$ and $\bar{F}$ is an algebraic closure of $F$.

Let $C(f) \subseteq \mathbf{R}^{p}$ be the real cone spanned by the elements of $J$, let $M(f)=\mathbf{Z}^{p} \cap C(f)$, and put $\hat{R}=F\left[x^{u} \mid u \in M(f)\right]$. Let $L(f) \subseteq \mathbf{R}^{p}$ be the 
real subspace spanned by the elements of $J$ and let $M^{\prime}(f)=\mathbf{Z}^{p} \cap L(f)$. Put $d(f)=\operatorname{dim}_{\mathbf{R}} L(f)$. Let $V(f)$ be the volume of $\Delta(f)$ relative to Lebesgue measure on $L(f)$ normalized so that a fundamental domain for the lattice $M^{\prime}(f)$ has volume 1 . Let $\alpha=\left(\alpha_{1}, \ldots, \alpha_{p}\right) \in F^{p}$ and define differential operators $D_{i, f, \alpha}$ on $\hat{R}$ for $i=1, \ldots, p$ by

$$
D_{i, f, \alpha}=x_{i} \frac{\partial}{\partial x_{i}}+\alpha_{i}+x_{i} \frac{\partial f}{\partial x_{i}} .
$$

Define a complex $\left(\hat{K}^{\cdot}, \delta_{f, \alpha}\right)$ by

$$
\begin{aligned}
\hat{K}^{l} & =\bigoplus_{1 \leq i_{1}<\cdots<i_{l} \leq p} \hat{R} \frac{d x_{i_{1}}}{x_{i_{1}}} \wedge \cdots \wedge \frac{d x_{i_{l}}}{x_{i_{l}}}, \\
\delta_{f, \alpha}\left(\xi \frac{d x_{i_{1}}}{x_{i_{1}}} \wedge \cdots \wedge \frac{d x_{i_{l}}}{x_{i_{l}}}\right) & =\left(\sum_{i=1}^{p} D_{i, f, \alpha}(\xi) \frac{d x_{i}}{x_{i}}\right) \wedge \frac{d x_{i_{1}}}{x_{i_{1}}} \wedge \cdots \wedge \frac{d x_{i_{l}}}{x_{i_{l}}} .
\end{aligned}
$$

Remark. Suppose we are in the situation of section 2 with $n=0$ and take $p=N+r, f=h$. The inclusion $\hat{R} \subseteq R^{\prime}$ identifies the complex $\left(\hat{K}^{\cdot}, \delta_{f, \alpha}\right)$ with a subcomplex of $\left(\Omega^{*}(* Z), \delta_{h, \alpha}\right)$. Our calculation of $H^{l}\left(\hat{K}^{*}\right)$ here will lead to a calculation of $H^{l}\left(\Omega^{*}(* Z)\right)$ in section 5 .

The subspace $L(f) \subseteq \mathbf{R}^{p}$ can be defined by linear equations with rational coefficients, i.e., there exists $L \subseteq \mathbf{Q}^{p}$ such that

$$
L(f)=\mathbf{R} \bigotimes_{\mathbf{Q}} L \subseteq \mathbf{R} \bigotimes_{\mathbf{Q}} \mathbf{Q}^{p}=\mathbf{R}^{p} .
$$

Our basic result is the following.

THEOREM 4.1. Suppose $f$ is nondegenerate relative to $\Delta(f)$. If $\alpha \notin$ $F \otimes_{\mathbf{Q}} L$, then $H^{l}\left(\hat{K}^{\cdot}\right)=0$ for all $l$. If $\alpha \in F \otimes_{\mathbf{Q}} L$, then $H^{l}\left(\hat{K}^{*}\right)=0$ for $l<d(f)$ or $l>p$ and $\operatorname{dim}_{F} H^{l}\left(\hat{K}^{\cdot}\right)=\left(\begin{array}{c}p-d(f) \\ l-d(f)\end{array}\right) d(f) ! V(f)$ for $d(f) \leq l \leq p$. In particular, if $d(f)=p$, then $H^{l}\left(\hat{K}^{\cdot}\right)=0$ if $l \neq p$ and $\operatorname{dim}_{F} H^{p}\left(\hat{K}^{*}\right)=$ $p ! V(f)$.

Proof. The case $\alpha \notin F \otimes_{\mathbf{Q}} L$ is essentially trivial. Choose a linear form $\rho=\sum_{i=1}^{p} b_{i} u_{i}$ on $\mathbf{Q}^{p}$ such that $\rho$ vanishes on $L$ but $\rho(\alpha) \neq 0$. Put

$$
\rho\left(D_{f, \alpha}\right)=\sum_{i=1}^{p} b_{i} D_{i, f, \alpha}
$$


a differential operator on $\hat{R}$. It commutes with the differential $\delta_{f, \alpha}$ of the complex $\hat{K}^{\cdot}$. Since $\hat{K}^{\cdot}$ is (up to reindexing and some sign changes) the Koszul complex on $\hat{R}$ defined by $\left\{D_{i, f, \alpha}\right\}_{i=1}^{p}$, it follows that $\rho\left(D_{f, \alpha}\right)$ is the zero operator on $H^{l}\left(\hat{K}^{*}\right)$ for all $l$. On the other hand, since all monomials $x^{u} \in \hat{R}$ satisfy $u \in L$, a calculation shows that $\rho\left(D_{f, \alpha}\right)$ acts on $\hat{R}$ by multiplication by $\rho(\alpha)$. Since $\rho(\alpha) \neq 0, \rho\left(D_{f, \alpha}\right)$ is invertible on $\hat{R}$ and hence on $H^{l}\left(\hat{K}^{*}\right)$ for all $l$. This implies $H^{l}\left(\hat{K}^{\cdot}\right)=0$ for all $l$.

From now on we assume $\alpha \in F \otimes_{\mathbf{Q}} L$. The ring $\hat{R}$ has an increasing filtration $F$. defined as follows. Define the weight $w(u)$ of $u \in M(f)$ to be the least nonnegative real (hence rational) number $w$ such that $u \in w \Delta(f)$, the dilation of $\Delta(f)$ by the factor $w$. It is easily seen that there exists a positive integer $e$ such that $w(u) \in e^{-1} \mathbf{Z}_{\geq 0}$. Define $F_{k / e} \hat{R}$ to be the $F$-span of those monomials $x^{u}$ with $w(u) \leq k / e$. Note that $x_{i} \partial f / \partial x_{i} \in F_{1} \hat{R}$ for $i=1, \ldots, p$. We let $\bar{f}_{i}$ denote the image of $x_{i} \partial f / \partial x_{i}$ in $\operatorname{gr}_{1}(\hat{R})$, where gr. $(\hat{R})$ is the associated graded ring.

This filtration induces a filtration on the complex $\hat{K}^{\cdot}$ by defining $F_{k / e} \hat{K}^{l}$ to be the span of the $l$-forms $x^{u}\left(d x_{i_{1}} / x_{i_{1}}\right) \wedge \cdots \wedge\left(d x_{i_{l}} / x_{i_{l}}\right)$ with $w(u) \leq$ $(k / e)-l$. The differential $\delta_{f, \alpha}$ preserves this filtration. We denote the associated graded complex by $\left(\bar{K}^{\cdot}, \bar{\delta}_{f, \alpha}\right)$. Explicitly,

$$
\begin{aligned}
& \bar{K}^{l}=\bigoplus_{1 \leq i_{1}<\cdots<i_{l} \leq p} \operatorname{gr} .(\hat{R}) \frac{d x_{i_{1}}}{x_{i_{1}}} \wedge \cdots \wedge \frac{d x_{i_{l}}}{x_{i_{l}}}, \\
& \bar{\delta}_{f, \alpha}\left(\xi \frac{d x_{i_{1}}}{x_{i_{1}}} \wedge \cdots \wedge \frac{d x_{i_{l}}}{x_{i_{l}}}\right)=\left(\sum_{i=1}^{p} \bar{f}_{i} \xi \frac{d x_{i}}{x_{i}}\right) \wedge \frac{d x_{i_{1}}}{x_{i_{1}}} \wedge \cdots \wedge \frac{d x_{i_{l}}}{x_{i_{l}}} .
\end{aligned}
$$

The subspace $L$ can be parametrized by $d(f)$ coordinates. To fix ideas, suppose these coordinates are $u_{1}, \ldots, u_{d(f)}$. Then $L$ can be defined by equations

$$
u_{i}=\sum_{j=1}^{d(f)} b_{i j} u_{j} \quad\left(i=d(f)+1, \ldots, p, b_{i j} \in \mathbf{Q}\right)
$$

Since the exponent of every monomial in $f$ lies in $L$, it follows that

$$
x_{i} \frac{\partial f}{\partial x_{i}}=\sum_{j=1}^{d(f)} b_{i j} x_{j} \frac{\partial f}{\partial x_{j}} \quad(i=d(f)+1, \ldots, p)
$$


in particular, we have

$$
\bar{f}_{i}=\sum_{j=1}^{d(f)} b_{i j} \bar{f}_{j} \quad(i=d(f)+1, \ldots, p) .
$$

Let $\left(\bar{K}_{1}, \bar{\delta}_{1}\right)$ be the complex defined using only $\bar{f}_{1}, \ldots, \bar{f}_{d(f)}$, i.e.,

$$
\begin{aligned}
\bar{K}_{1}^{l} & =\bigoplus_{1 \leq i_{1}<\cdots<i_{l} \leq d(f)} \operatorname{gr} .(\hat{R}) \frac{d x_{i_{1}}}{x_{i_{1}}} \wedge \cdots \wedge \frac{d x_{i_{l}}}{x_{i_{l}}}, \\
\bar{\delta}_{1}\left(\xi \frac{d x_{i_{1}}}{x_{i_{1}}} \wedge \cdots \wedge \frac{d x_{i_{l}}}{x_{i_{l}}}\right) & =\left(\sum_{i=1}^{d(f)} \bar{f}_{i} \xi \frac{d x_{i}}{x_{i}}\right) \wedge \frac{d x_{i_{1}}}{x_{i_{1}}} \wedge \cdots \wedge \frac{d x_{i_{l}}}{x_{i_{l}}} .
\end{aligned}
$$

By [KO Théorème 2.8] (see also [AS3 Theorem 2.14]), the hypothesis that $f$ is nondegenerate implies that

$$
H^{l}\left(\bar{K}_{1}\right)=0 \quad(l \neq d(f))
$$

and $\operatorname{dim}_{F} H^{d(f)}\left(\bar{K}_{1}^{*}\right)=d(f) ! V(f)$.

Let $\left(\hat{K}_{1}^{\cdot}, \delta_{1}\right)$ be the corresponding complex defined by $D_{i, f, \alpha}$ for $i=$ $1, \ldots, d(f)$, i.e.,

$$
\begin{gathered}
\hat{K}_{1}^{l}=\bigoplus_{1 \leq i_{1}<\cdots<i_{l} \leq d(f)} \hat{R} \frac{d x_{i_{1}}}{x_{i_{1}}} \wedge \cdots \wedge \frac{d x_{i_{l}}}{x_{i_{l}}}, \\
\delta_{1}\left(\xi \frac{d x_{i_{1}}}{x_{i_{1}}} \wedge \cdots \wedge \frac{d x_{i_{l}}}{x_{i_{l}}}\right)=\left(\sum_{i=1}^{d(f)} D_{i, f, \alpha}(\xi) \frac{d x_{i}}{x_{i}}\right) \wedge \frac{d x_{i_{1}}}{x_{i_{1}}} \wedge \cdots \wedge \frac{d x_{i_{l}}}{x_{i_{l}}} .
\end{gathered}
$$

This is a filtered complex (using the previously defined filtration) whose associated graded complex is $\bar{K}_{1}^{*}$. Thus there is an $E_{1}$ spectral sequence whose $E_{1}$ term is the cohomology of $\bar{K}_{1}^{j}$ and whose $E_{\infty}$ term is the associated graded of the cohomology of $\hat{K}_{1}$. By (4.3) this spectral sequence collapses at the $E_{1}$ term, hence

$$
\begin{aligned}
H^{l}\left(\hat{K}_{1}^{*}\right) & =0 \\
\operatorname{dim}_{F} H^{d(f)}\left(\hat{K}_{1}^{*}\right) & =d(f) ! V(f) .
\end{aligned} \quad(l \neq d(f)),
$$

Since $\alpha \in F \otimes_{\mathbf{Q}} L$, as operators on $\hat{R}$ we have

$$
D_{i, f, \alpha}=\sum_{j=1}^{d(f)} b_{i j} D_{j, f, \alpha} \quad(i=d(f)+1, \ldots, p) .
$$


By standard properties of complexes, (4.4) implies

$$
H^{l}\left(\hat{K}^{\cdot}\right)=H^{l-d(f)}\left(\hat{K}_{2}^{\cdot}\right)
$$

where $\hat{K}_{2}^{\cdot}$ is the complex

$$
\begin{array}{r}
\hat{K}_{2}^{l}=\bigoplus_{d(f)+1 \leq i_{1}<\cdots<i_{l} \leq p} H^{d(f)}\left(\hat{K}_{1}^{*}\right) \frac{d x_{i_{1}}}{x_{i_{1}}} \wedge \cdots \wedge \frac{d x_{i_{l}}}{x_{i_{l}}} \\
\delta_{2}\left(\xi \frac{d x_{i_{1}}}{x_{i_{1}}} \wedge \cdots \wedge \frac{d x_{i_{l}}}{x_{i_{l}}}\right)=\left(\sum_{i=d(f)+1}^{p} D_{i, f, \alpha}(\xi) \frac{d x_{i}}{x_{i}}\right) \wedge \frac{d x_{i_{1}}}{x_{i_{1}}} \wedge \cdots \wedge \frac{d x_{i_{l}}}{x_{i_{l}}}
\end{array}
$$

But it follows from (4.6) that $D_{i, f, \alpha}$ acts trivially on $H^{d(f)}\left(\hat{K}_{1}^{\cdot}\right)$ for $i=$ $d(f)+1, \ldots, p$, hence the differential $\delta_{2}$ is trivial. It is then clear that $H^{l}\left(\hat{K}_{2}^{*}\right)$ is isomorphic to the direct sum of $\left(\begin{array}{c}p-d(f) \\ l\end{array}\right)$ copies of $H^{d(f)}\left(\hat{K}_{1}^{*}\right)$. Theorem 4.1 now follows from (4.5) and (4.7).

We now define some complexes "between" $\hat{K}^{\cdot}$ and $\Omega^{*}(* Z)$. Let $\sigma_{1}, \ldots$, $\sigma_{s}$ be the codimension-one faces of the cone $C(f)$. Define linear forms $l_{1}, \ldots, l_{s}$ on $L$ by the conditions: (i) $l_{i}=0$ on $\sigma_{i}$, (ii) $l_{i}\left(M^{\prime}(f)\right)=\mathbf{Z}$, (iii) $l_{i} \geq 0$ on $C(f)$. It is easily checked that for $u \in L(f), u \in C(f)$ if and only if $l_{i}(u) \geq 0$ for $i=1, \ldots, s$. Let $I \subseteq\{1, \ldots, s\}$. We say that $\alpha$ is semi-nonresonant relative to $I$ if either $\alpha \notin F \otimes_{\mathbf{Q}} L$ or $l_{i}(\alpha)$ is not a positive integer for $i \in I$. For $I \subseteq\{1, \ldots, s\}$, let $M_{I}(f)=\left\{u \in M^{\prime}(f) \mid\right.$ $l_{i}(u) \geq 0$ for all $\left.i \notin I\right\}$ and let $R_{I}$ be the ring $R_{I}=F\left[x^{u} \mid u \in M_{I}(f)\right]$. In particular, $M_{\emptyset}(f)=M(f)$ and $R_{\emptyset}=\hat{R}$. Define a complex $\left(K_{I}^{\cdot}, \delta_{f, \alpha}\right)$ by

$$
\begin{gathered}
K_{I}^{l}=\bigoplus_{1 \leq i_{1}<\cdots<i_{l} \leq p} R_{I} \frac{d x_{i_{1}}}{x_{i_{1}}} \wedge \cdots \wedge \frac{d x_{i_{l}}}{x_{i_{l}}} \\
\delta_{f, \alpha}\left(\xi \frac{d x_{i_{1}}}{x_{i_{1}}} \wedge \cdots \wedge \frac{d x_{i_{l}}}{x_{i_{l}}}\right)=\left(\sum_{i=1}^{p} D_{i, f, \alpha}(\xi) \frac{d x_{i}}{x_{i}}\right) \wedge \frac{d x_{i_{1}}}{x_{i_{1}}} \wedge \cdots \wedge \frac{d x_{i_{l}}}{x_{i_{l}}} .
\end{gathered}
$$

The natural inclusion $\hat{R} \hookrightarrow R_{I}$ identifies $\left(\hat{K}^{\cdot}, \delta_{f, \alpha}\right)$ with a subcomplex of $\left(K_{I}^{\dot{1}}, \delta_{f, \alpha}\right)$.

Proposition 4.8. If $\alpha$ is semi-nonresonant relative to $I \subseteq\{1, \ldots, s\}$, then the inclusion $\left(\hat{K}^{\cdot}, \delta_{f, \alpha}\right) \hookrightarrow\left(K_{I}^{*}, \delta_{f, \alpha}\right)$ is a quasi-isomorphism, i.e., it induces isomorphisms $H^{l}\left(\hat{K}^{\cdot}\right) \simeq H^{l}\left(K_{I}^{*}\right)$ for all $l$. 
Proof. When $\alpha \notin F \bigotimes_{\mathbf{Q}} L$, the same argument that was used in the proof of Theorem 4.1 for $\hat{K}^{\cdot}$ shows that $H^{l}\left(K_{I}^{*}\right)=0$ for all $l$. So we may assume that $\alpha \in F \bigotimes_{\mathbf{Q}} L$. Note that if $\alpha$ is semi-nonresonant relative to $I$ then $\alpha$ is semi-nonresonant relative to $J$ for every subset $J \subseteq I$. It clearly suffices to show that for every $J \subseteq I$ and $i \in I \backslash J$, the natural inclusion $\left(K_{J}^{\cdot}, \delta_{f, \alpha}\right) \hookrightarrow\left(K_{J \cup\{i\}}^{\cdot}, \delta_{f, \alpha}\right)$ is a quasi-isomorphism. We achieve this by showing that the quotient complex $K_{J \cup\{i\}}^{\cdot} / K_{J}^{\cdot}$ satisfies $H^{l}\left(K_{J \cup\{i\}}^{\cdot} / K_{J}^{*}\right)=0$ for all $l$. We view this quotient complex as follows. The quotient $R_{J \cup\{i\}} / R_{J}$ may be identified with the $F$-vector space $V$ with basis the set of all $x^{u}$ for $u \in M^{\prime}(f)$ satisfying $l_{k}(u) \geq 0$ for $k \notin J \cup\{i\}, l_{i}(u)<0$. We then have the identifications

$$
\begin{gathered}
\left(K_{J \cup\{i\}}^{\cdot} / K_{J}^{*}\right)^{l}=\bigoplus_{1 \leq i_{1}<\cdots<i_{l} \leq p} V \frac{d x_{i_{1}}}{x_{i_{1}}} \wedge \cdots \wedge \frac{d x_{i_{l}}}{x_{i_{l}}} \\
\delta_{f, \alpha}\left(\xi \frac{d x_{i_{1}}}{x_{i_{1}}} \wedge \cdots \wedge \frac{d x_{i_{l}}}{x_{i_{l}}}\right)=\left(\sum_{k=1}^{p} \gamma_{-}\left(D_{k, f, \alpha}(\xi)\right) \frac{d x_{k}}{x_{k}}\right) \wedge \frac{d x_{i_{1}}}{x_{i_{1}}} \wedge \cdots \wedge \frac{d x_{i_{l}}}{x_{i_{l}}}
\end{gathered}
$$

where

$$
\gamma_{-}\left(x^{u}\right)= \begin{cases}x^{u} & \text { if } x^{u} \in V, \\ 0 & \text { if } x^{u} \notin V .\end{cases}
$$

Thus the quotient complex can be identified with the Koszul complex on $V$ defined by the operators $\left\{\gamma_{-} \circ D_{k, f, \alpha}\right\}_{k=1}^{p}$.

There is an increasing filtration $F$. on $V$ defined by letting $F_{a} V$ be the subspace spanned by those $x^{u}$ with $l_{i}(u) \geq-a, a=0,1, \ldots$. Note that $F_{0} V=(0)$. By a standard spectral sequence argument, it suffices to show that the associated graded complex has trivial cohomology. We identify the associated graded complex with the Koszul complex on $\operatorname{gr} .(V)$ defined by the induced action of $\gamma_{-} \circ D_{k, f, \alpha}, k=1, \ldots, p$, which preserve this filtration. Extend the form $l_{i}$ on $L$ to $\mathbf{Q}^{p}$ and write it as

$$
l_{i}\left(u_{1}, \ldots, u_{p}\right)=\sum_{k=1}^{p} c_{k} u_{k} \quad\left(c_{k} \in \mathbf{Q}\right) .
$$

Put $l_{i}\left(D_{f, \alpha}\right)=\sum_{k=1}^{p} c_{k} \gamma_{-} \circ D_{k, f, \alpha}$, an operator on $V$. One checks that it is independent of the choice of extension of $l_{i}$ to $\mathbf{Q}^{p}$. Then $l_{i}\left(D_{f, \alpha}\right)$ is the zero operator on all homology groups of the Koszul complex on gr. $(V)$ defined by $\left\{\gamma_{-} \circ D_{k, f, \alpha}\right\}_{k=1}^{p}$. We show that $l_{i}\left(D_{f, \alpha}\right)$ is invertible on gr. $(V)$, which implies that all these homology groups must vanish. 
One computes that

$$
l_{i}\left(D_{f, \alpha}\right)\left(x^{u}\right)=l_{i}(\alpha+u) x^{u}+\gamma_{-}\left(\sum_{j} a_{j} l_{i}(j) x^{j+u}\right)
$$

(recall $\left.f=\sum_{j} a_{j} x^{j}\right)$. But $l_{i}(j) \geq 0$, so each $x^{j+u}$ that appears in (4.9) with nonzero coefficient satisfies $l_{i}(j+u)>l_{i}(u)$. It then follows from (4.9) that $l_{i}\left(D_{f, \alpha}\right)$ operates on $\operatorname{gr}_{a}(V)$ as multiplication by $l_{i}(\alpha)-a$. The hypothesis that $\alpha$ be semi-nonresonant implies this is nonzero (with the possible exception of the case $a=0$, which is not a problem since $\operatorname{gr}_{0}(V)=$ $(0))$.

From Theorem 4.1 and Proposition 4.8, we have immediately the following.

COROLlARY 4.10. Suppose that $f$ is nondegenerate relative to $\Delta(f)$ and $\alpha$ is semi-nonresonant relative to $I \subseteq\{1, \ldots, s\}$. If $\alpha \notin F \bigotimes_{\mathbf{Q}} L$, then $H^{l}\left(K_{I}^{*}\right)=0$ for all $l$. If $\alpha \in F \otimes_{\mathbf{Q}} L$, then $H^{l}\left(K_{I}^{*}\right)=0$ for $l<d(f)$ or $l>p$ and $\operatorname{dim}_{F} H^{l}\left(K_{I}^{*}\right)=\left(\begin{array}{l}p-d(f) \\ l-d(f)\end{array}\right) d(f) ! V(f)$ for $d(f) \leq l \leq p$.

In the special case $I=\Sigma \stackrel{\text { def }}{=}\{1, \ldots, s\}$, we can drop the hypothesis of semi-nonresonancy. This follows because for any $u \in M^{\prime}(f)$, multiplication by $x^{u}$ is an isomorphism of complexes

$$
\left(K_{\Sigma}^{\cdot}, \delta_{f, \alpha}\right) \simeq\left(K_{\Sigma}^{\cdot}, \delta_{f, \alpha-u}\right)
$$

(its inverse is multiplication by $x^{-u}$ ). Even if $\alpha$ fails to be semi-nonresonant relative to $\Sigma$, we can always choose $u \in M(f)$ so that $\alpha-u$ is seminonresonant relative to $\Sigma$ and apply Proposition 4.8 to the complex on the right-hand side. Thus we have the following.

Corollary 4.11. Suppose $f$ is nondegenerate relative to $\Delta(f)$. If $\alpha \notin F \otimes_{\mathbf{Q}} L$, then $H^{l}\left(K_{\Sigma}\right)=0$ for all $l$. If $\alpha \in F \otimes_{\mathbf{Q}} L$, then $H^{l}\left(K_{\Sigma}^{*}\right)=0$ for $l<d(f)$ or $l>p$ and $\operatorname{dim}_{F} H^{l}\left(K_{\{1, \ldots, s\}}^{\cdot}\right)=\left(\begin{array}{l}p-d(f) \\ l-d(f)\end{array}\right) d(f) ! V(f)$ for all $d(f) \leq l \leq p$.

Put $R_{0}=F\left[x_{1}, \ldots, x_{p},\left(x_{1} \cdots x_{p}\right)^{-1}\right]$. We compute the cohomology of the complex $K_{0}$ defined by

$$
\begin{aligned}
K_{0}^{l} & =\bigoplus_{1 \leq i_{1}<\cdots<i_{l} \leq p} R_{0} \frac{d x_{i_{1}}}{x_{i_{1}}} \wedge \cdots \wedge \frac{d x_{i_{l}}}{x_{i_{l}}} \\
\delta_{f, \alpha}\left(\xi \frac{d x_{i_{1}}}{x_{i_{1}}} \wedge \cdots \wedge \frac{d x_{i_{l}}}{x_{i_{l}}}\right) & =\left(\sum_{i=1}^{p} D_{i, f, \alpha}(\xi) \frac{d x_{i}}{x_{i}}\right) \wedge \frac{d x_{i_{1}}}{x_{i_{1}}} \wedge \cdots \wedge \frac{d x_{i_{l}}}{x_{i_{l}}} .
\end{aligned}
$$


Note that $R_{0}$ has a direct sum decomposition as $F$-vector space

$$
R_{0}=\bigoplus_{[u] \in \mathbf{Z}^{p} / M^{\prime}(f)} R_{0}^{[u]}
$$

where $R_{0}^{[u]}$ is the $F$-span of all $x^{v}$ such that $v \in[u]$. Each $D_{i, f, \alpha}$ is stable on $R_{0}^{[u]}$, hence we get a corresponding direct sum decomposition

$$
\left(K_{0}^{\cdot}, \delta_{f, \alpha}\right)=\bigoplus_{[u] \in \mathbf{Z}^{p} / M^{\prime}(f)}\left(K_{0,[u]}^{\cdot}, \delta_{f, \alpha}\right)
$$

When $u \in M^{\prime}(f),\left(K_{0,[u]}^{\cdot}, \delta_{f, \alpha}\right)$ is just the above complex $\left(K_{\Sigma}^{\cdot}, \delta_{f, \alpha}\right)$. Furthermore, for $u \in \mathbf{Z}^{p}$, one checks easily that

$$
D_{i, f, \alpha} \circ x^{u}=x^{u} \circ D_{i, f, \alpha+u},
$$

which says that multiplication by $x^{u}$ is an isomorphism of complexes between $\left(K_{\Sigma}^{*}, \delta_{f, \alpha+u}\right)$ and $\left(K_{0,[u]}^{\cdot}, \delta_{f, \alpha}\right)$. Thus by Corollary $4.11, H^{l}\left(K_{0,[u]}^{\cdot}\right.$, $\left.\delta_{f, \alpha}\right)=0$ for all $l$ if $\alpha+u \notin F \bigotimes_{\mathbf{Q}} L$ while if $\alpha+u \in F \bigotimes_{\mathbf{Q}} L$, then $H^{l}\left(K_{0,[u]}^{\cdot}, \delta_{f, \alpha}\right)=0$ for $l<d(f)$ or $l>p$ and $\operatorname{dim}_{F} H^{l}\left(K_{0,[u]}^{\cdot}, \delta_{f, \alpha}\right)=$ $\left(\begin{array}{l}p-d(f) \\ l-d(f)\end{array}\right) d(f) ! V(f)$ for $d(f) \leq l \leq p$. There is either zero or one class $[u] \in \mathbf{Z}^{p} / M^{\prime}(f)$ such that $\alpha+u \in F \otimes_{\mathbf{Q}} L$, according as to whether $\left(\alpha+\mathbf{Z}^{p}\right) \cap\left(F \bigotimes_{\mathbf{Q}} L\right)$ is empty or nonempty. By $(4.12)$, we therefore have the following.

THEOREM 4.13. Suppose $f$ is nondegenerate. If $\left(\alpha+\mathbf{Z}^{p}\right) \cap\left(F \otimes_{\mathbf{Q}} L\right)=$ $\emptyset$, then $H^{l}\left(K_{0}^{\cdot}, \delta_{f, \alpha}\right)=0$ for all l. If $\left(\alpha+\mathbf{Z}^{p}\right) \cap\left(F \otimes_{\mathbf{Q}} L\right) \neq \emptyset$, then $H^{l}\left(K_{0}^{\cdot}, \delta_{f, \alpha}\right)=0$ for $l<d(f)$ or $l>p$ and

$$
\operatorname{dim}_{F} H^{l}\left(K_{0}^{\cdot}, \delta_{f, \alpha}\right)=\left(\begin{array}{l}
p-d(f) \\
l-d(f)
\end{array}\right) d(f) ! V(f)
$$

for $d(f) \leq l \leq p$.

\section{$\S 5$. Proof of Theorem $\mathbf{1 . 4}$}

We return to the setting of sections 2 and 3 but make the additional assumption that we are in the purely toric case, i.e., that $n=0$. Thus

$$
\begin{gathered}
g, f_{1}, \ldots, f_{r} \in F\left[x_{1}, \ldots, x_{N},\left(x_{1} \cdots x_{N}\right)^{-1}\right] \\
h=g+\sum_{j=1}^{r} x_{N+j} f_{j} \in F\left[x_{1}, \ldots, x_{N+r},\left(x_{1} \cdots x_{N}\right)^{-1}\right] .
\end{gathered}
$$


THEOREM 5.1. Suppose $h$ is nondegenerate relative to $\Delta(h)$ and $\operatorname{dim} \Delta(h)=N+r$. Then

$$
\begin{aligned}
H^{l}\left(\Omega^{*}(* Z), \delta_{h, \alpha}\right) & =0 & (l \neq N+r), \\
\operatorname{dim}_{F} H^{N+r}\left(\Omega^{*}(* Z), \delta_{h, \alpha}\right) & =(N+r) ! V(h) . &
\end{aligned}
$$

Proof. We apply the results of section 4, taking $p=N+r, f=$ $h$. One checks that the complex $\left(K_{0}^{*}, \delta_{f, \alpha}\right)$ of section 4 then becomes $\left(\Omega^{*}(* Z), \delta_{h, \alpha}\right)$. Since $\operatorname{dim} \Delta(h)=N+r$, we have $L=\mathbf{Q}^{N+r}$. Thus $\left(\alpha+\mathbf{Z}^{N+r}\right) \cap\left(F \otimes_{\mathbf{Q}} \mathbf{Q}^{N+r}\right) \neq \emptyset$ for all $\alpha$ and $d(f)=N+r$. The theorem is then an immediate consequence of Theorem 4.13.

Proof of Theorem 1.4. By hypothesis, $\alpha_{N+1}, \ldots, \alpha_{N+r} \notin \mathbf{Z}, h$ is nondegenerate relative to $\Delta(h)$, and $\operatorname{dim} \Delta(h)=N+r$. Suppose we can show that these conditions imply the hypothesis of Proposition 3.1, namely, that $H^{l}\left(A_{\emptyset}^{*}\right)=0$ for $l \neq r$. Then by Proposition 3.1 and Theorem 2.8 we have isomorphisms

$$
H^{l+r}\left(\Omega^{\cdot}(* Z)\right) \simeq H^{l}\left(\tilde{\Omega}^{\cdot}(* Z)\right) \simeq H^{l}\left(\Omega^{\cdot}(* Y)\right)
$$

for all $l$. Theorem 1.4 is then an immediate consequence of Theorem 5.1. We are thus reduced to checking that $H^{l}\left(A_{\emptyset}\right)=0$ for $l \neq r$.

As observed in the remark following Proposition 3.1, the complex $A_{\emptyset}$ is (up to reindexing and some sign changes) the Koszul complex on $R^{\prime}=$ $F\left[x_{1}, \ldots, x_{N+r},\left(x_{1} \cdots x_{N+r}\right)^{-1}\right]$ defined by the operators $\left\{D_{N+j, h, \alpha}\right\}_{j=1}^{r}$. We denote this Koszul complex by $K$. in what follows. Thus we must check that $K$. is acyclic in positive dimension, i.e.,

$$
H_{l}(K .)=0 \quad \text { for } l>0 \text {. }
$$

We accomplish this by a modification of the arguments of section 4 .

Note that $D_{N+j, h, \alpha}=x_{N+j} \partial / \partial x_{N+j}+\alpha_{N+j}+x_{N+j} f_{j}$ is independent of $g$. Furthermore, the nondegeneracy of $h$ relative to $\Delta(h)$ implies the nondegeneracy of $\sum_{j=1}^{r} x_{N+j} f_{j}$ relative to $\Delta\left(\sum_{j=1}^{r} x_{N+j} f_{j}\right)$. (Every face $\sigma$ of $\Delta\left(\sum_{j=1}^{r} x_{N+j} f_{j}\right)$ not containing the origin is also a face of $\Delta(h)$ and $\left(\sum_{j=1}^{r} x_{N+j} f_{j}\right)_{\sigma}=h_{\sigma}$ for such a face.) So we may assume $g=0$, i.e., $h=\sum_{j=1}^{r} x_{N+j} f_{j}$. The ring $\hat{R}$ of section 4 is generated by monomials $x^{u}$ with $u \in \mathbf{Z}^{N+r} \cap C(h)$. The weight function $w$ defined in section 4 is given explicitly in this case by $w(u)=u_{N+1}+\cdots+u_{N+r}$, and it defines a grading (not just a filtration) on $\hat{R}$. Furthermore, $\hat{R}$ is known to be 
a Cohen-Macaulay ring (see $[\mathrm{H}]$ ). The theorem of Kouchnirenko takes a sharper form in this case (see [KO section 2.12]). The hypothesis that $h$ is nondegenerate and $\operatorname{dim} \Delta(h)=N+r$ imply that $\left\{x_{i} \partial h / \partial x_{i}\right\}_{i=1}^{N+r}$ form a regular sequence on $\hat{R}$. Taking $i=N+1, \ldots, N+r$, we see in particular that $\left\{x_{N+j} f_{j}\right\}_{j=1}^{r}$ form a regular sequence on $\hat{R}$, hence the Koszul complex they define on $\hat{R}$ is acyclic in positive dimension. Fix an element $u \in M(h)$ that is also an interior point of $C(h)$. For each integer $a \geq 0$, let $C^{(a)}$ be the Koszul complex on the $\hat{R}$-module $x^{-a u} \hat{R}$ defined by $\left\{x_{N+j} f_{j}\right\}_{j=1}^{r}$. Since multiplication by $x^{-a u}$ is an $\hat{R}$-module isomorphism from $\hat{R}$ onto $x^{-a u} \hat{R}$, it follows that $H_{l}\left(C^{(a)}\right)=0$ for all $l>0$ and all $a$. Let $D^{(a)}$ be the Koszul complex on $x^{-a u} \hat{R}$ defined by $\left\{D_{N+j, h, \alpha}\right\}_{j=1}^{r}$. This is a filtered complex, where the filtration $F$. on $x^{-a u} \hat{R}$ is defined by taking $F_{k}\left(x^{-a u} \hat{R}\right)$ to be the $F$-span of all $x^{-a u} x^{v}$ with $w(v) \leq k$. Its associated graded complex is $C{ }^{(a)}$, hence the same spectral sequence argument used to prove (4.4) shows that $H_{l}\left(D^{(a)}\right)=0$ for all $l>0$ and all $a$.

Now consider $K$., the Koszul complex defined above. Since $R^{\prime}=$ $\bigcup_{a=0}^{\infty} x^{-a u} \hat{R}$, it follows that any l-cycle $\xi$ representing a homology class of $H_{l}(K$.$) is an l$-cycle in some complex $D^{(a)}$. But $H_{l}\left(D^{(a)}\right)=0$ for $l>0$, i.e., $\xi$ is an $l$-boundary in $D^{(a)}$, hence $\xi$ is an $l$-boundary in $K$. also. It follows that $H_{l}(K)=$.0 for all $l>0$. This establishes (5.2).

\section{$\S$ 6. Cohomology of $\Omega^{\cdot}(* Z)$ on $\mathbf{T}^{m} \times \mathbf{A}^{n+r}$}

In this section we go beyond the purely toric case and consider the problem of computing the cohomology of $\Omega^{*}(* Z)$ on $\mathbf{T}^{m} \times \mathbf{A}^{n+r}$. For this it is necessary to pursue the ideas of section 4 a little further. For most of this section, we deal with an arbitrary polynomial $h \in F\left[x_{1}, \ldots, x_{N+r},\left(x_{1} \ldots\right.\right.$ $\left.x_{m}\right)^{-1}$. For the application to twisted de Rham cohomology (Theorem 6.7 below), we shall take $h$ to be as given in (2.2).

For any subset $I \subseteq S$ with $S_{\text {to }} \cup S_{\text {du }} \subseteq I$, let $h_{I}$ be the polynomial obtained from $h$ by setting $x_{j}=0$ for $j \notin I$. (Note that $j \notin I$ implies $j \in S_{\mathrm{af}} \cap S_{\mathrm{sp}}$.) We say that $h$ is convenient relative to $S_{\mathrm{af}} \cap S_{\mathrm{sp}}$ if for all such $I$ we have

$$
\operatorname{dim} \Delta\left(h_{I}\right)=|I|
$$

This implies in particular that $\operatorname{dim} \Delta(h)=N+r$.

Let $l_{1}, \ldots, l_{s}$ be the linear forms on $\mathbf{Q}^{N+r}$ defining the codimensionone faces of the cone $C(h)$, normalized as in section 4 . The hypothesis that $h$ be convenient relative to $S_{\mathrm{af}} \cap S_{\mathrm{sp}}$ implies that the equations $x_{i}=$ 
0 for $i=m+1, \ldots, m+n$ define codimension-one faces of $C(h)$, hence must lie among the forms $l_{1}, \ldots, l_{s}$, say, $l_{1}=x_{m+1}, \ldots, l_{n}=x_{m+n}$. Let $B=\{n+1, \ldots, s\} \subseteq\{1, \ldots, s\}$. We recall the corresponding complex $K_{B}$ defined immediately preceding Proposition 4.8. We have $M^{\prime}(h)=\mathbf{Z}^{N+r}$, hence

$$
M_{B}(h)=\left\{u=\left(u_{1}, \ldots, u_{N+r}\right) \in \mathbf{Z}^{N+r} \mid u_{m+1}, \ldots, u_{m+n} \geq 0\right\} .
$$

It is then clear that the $\operatorname{ring} R_{B}=F\left[x^{u} \mid u \in M_{B}(h)\right]$ defined there is identical to the ring $R^{\prime}=\Omega^{0}(* Z)$. Thus $\left(K_{B}^{\cdot}, \delta_{h, \alpha}\right)$ is the complex

$$
\begin{aligned}
& K_{B}^{l}=\bigoplus_{1 \leq i_{1}<\cdots<i_{l} \leq N+r} R^{\prime} \frac{d x_{i_{1}}}{x_{i_{1}}} \wedge \cdots \wedge \frac{d x_{i_{l}}}{x_{i_{l}}}, \\
& \delta\left(\xi \frac{d x_{i_{1}}}{x_{i_{1}}} \wedge \cdots \wedge \frac{d x_{i_{l}}}{x_{i_{l}}}\right)=\left(\sum_{i=1}^{N+r} D_{i, h, \alpha}(\xi) \frac{d x_{i}}{x_{i}}\right) \wedge \frac{d x_{i_{1}}}{x_{i_{1}}} \wedge \cdots \wedge \frac{d x_{i_{l}}}{x_{i_{l}}} .
\end{aligned}
$$

Note that $\Omega^{\cdot}(* Z)$ is a subcomplex of $K_{B}^{\cdot}$. More precisely, the difference between these two complexes is that in $K_{B}^{\circ}$ we allow logarithmic poles along the divisor $\prod_{i=1}^{n} x_{m+i}=0$ whereas in $\Omega^{\cdot}(* Z)$ we do not.

Proposition 6.2. Suppose that $h$ is nondegenerate relative to $\Delta(h)$ and convenient relative to $S_{\mathrm{af}} \cap S_{\mathrm{sp}}$ and that $\alpha$ is semi-nonresonant relative to $B$. Then

$$
\begin{aligned}
H^{l}\left(K_{B}^{*}, \delta_{h, \alpha}\right) & =0 & (l \neq N+r), \\
\operatorname{dim}_{F} H^{N+r}\left(K_{B}^{*}, \delta_{h, \alpha}\right) & =(N+r) ! V(h) . &
\end{aligned}
$$

Proof. The proposition is a special case of Corollary 4.10 .

We explain how to drop the hypothesis that $\alpha$ be semi-nonresonant. A short computation shows that the condition that $h$ be convenient implies that there exists $u \in C(h)$ with $l_{i}(u)=0$ for $i=1, \ldots, n$ but $l_{i}(u)>0$ for $i \in B$. In particular, both $x^{u}$ and $x^{-u}$ lie in $R^{\prime}$, so multiplication by $x^{u}$ is an isomorphism of complexes

$$
\left(K_{B}^{*}, \delta_{h, \alpha}\right) \simeq\left(K_{B}^{*}, \delta_{h, \alpha-u}\right) .
$$

The condition that $l_{i}(u)>0$ for $i \in B$ implies that $\alpha-k u$ is seminonresonant relative to $B$ when $k$ is a sufficiently large positive integer. Hence we get the following corollary. 
COROllary 6.3. The conclusion of Proposition 6.2 remains true when the hypothesis that $\alpha$ be semi-nonresonant relative to $B$ is dropped.

We connect $\left(K_{B}^{*}, \delta_{h, \alpha}\right)$ with $\left(\Omega^{*}(* Z), \delta_{h, \alpha}\right)$ by introducing some related complexes. For $I \subseteq S$ with $S_{\text {to }} \cup S_{\mathrm{du}} \subseteq I$, let

$$
R^{\prime}(I)=F\left[x^{u} \mid u \in M_{B}(h), u_{j}=0 \text { for } j \notin I\right],
$$

i.e., $R^{\prime}(I)$ is the coordinate ring of the variety which is the projection of $\mathbf{T}^{m} \times \mathbf{A}^{n} \times \mathbf{T}^{r}$ onto those coordinates which are indexed by $I$. For $i \in I$, let $D_{i, h_{I}, \alpha}$ be the differential operator on $R^{\prime}(I)$ defined by

$$
D_{i, h_{I}, \alpha}=x_{i} \frac{\partial}{\partial x_{i}}+\alpha_{i}+x_{i} \frac{\partial h_{I}}{\partial x_{i}} .
$$

Let $\left(K_{B}^{\cdot}(I), \delta_{h_{I}, \alpha}\right)$ be the complex defined by

$$
K_{B}^{l}(I)=\bigoplus R^{\prime}(I) \frac{d x_{i_{1}}}{x_{i_{1}}} \wedge \cdots \wedge \frac{d x_{i_{l}}}{x_{i_{l}}},
$$

where the direct sum is over all increasing sequences $i_{1}<\cdots<i_{l}$ of elements of $I$ and

$$
\delta_{h_{I}, \alpha}\left(\xi \frac{d x_{i_{1}}}{x_{i_{1}}} \wedge \cdots \wedge \frac{d x_{i_{l}}}{x_{i_{l}}}\right)=\left(\sum_{i \in I} D_{i, h_{I}, \alpha}(\xi) \frac{d x_{i}}{x_{i}}\right) \wedge \frac{d x_{i_{1}}}{x_{i_{1}}} \wedge \cdots \wedge \frac{d x_{i_{l}}}{x_{i_{l}}}
$$

For $I^{\prime} \subseteq I_{\mathrm{sp}}^{\mathrm{af}}$, let $R^{\prime}\left(I, I^{\prime}\right)=\left(\prod_{j \in I^{\prime}} x_{j}\right) R^{\prime}(I)$, the elements of $R^{\prime}(I)$ divisible by $x_{j}$ for all $j \in I^{\prime}$. Define a subcomplex $\left(K_{B}^{*}\left(I, I^{\prime}\right), \delta_{h_{I}, \alpha}\right)$ of $\left(K_{B}^{*}(I), \delta_{h_{I}, \alpha}\right)$ by

$$
K_{B}^{l}\left(I, I^{\prime}\right)=\bigoplus_{J \subseteq I,|J|=l} R^{\prime}\left(I, I^{\prime} \cap J\right) \frac{d x_{i_{1}}}{x_{i_{1}}} \wedge \cdots \wedge \frac{d x_{i_{l}}}{x_{i_{l}}}
$$

where $J=\left\{i_{1}, \ldots, i_{l}\right\}, i_{1}<\cdots<i_{l}$. Note that $K_{B}^{\cdot}(S, \emptyset)=K_{B}^{\cdot}(S)=K_{B}$ and $K_{B}\left(S, S_{\mathrm{af}} \cap S_{\mathrm{sp}}\right)=\Omega^{*}(* Z)$. Let $i \in I_{\mathrm{sp}}^{\mathrm{af}}, i \notin I^{\prime}$. Consider the map $\theta_{i}$ : $R^{\prime}\left(I, I^{\prime}\right) \rightarrow R^{\prime}\left(I \backslash\{i\}, I^{\prime}\right)$ defined by setting $x_{i}$ equal to 0 . Let $\tilde{K}_{B}^{\cdot}\left(I, I^{\prime}\right)$ be the complex obtained by shifting indices by 1 in $K_{B}^{\cdot}\left(I, I^{\prime}\right)$, i.e., $\tilde{K}_{B}^{l}\left(I, I^{\prime}\right)=$ $K_{B}^{l-1}\left(I, I^{\prime}\right)$. The map $\theta_{i}$ induces a map $\theta_{i}: K_{B}^{\cdot}\left(I, I^{\prime}\right) \rightarrow \tilde{K}_{B}^{\cdot}\left(I \backslash\{i\}, I^{\prime}\right)$ defined by

$$
\begin{aligned}
& \theta_{i}\left(\xi \frac{d x_{i_{1}}}{x_{i_{1}}} \wedge \cdots \wedge \frac{d x_{i_{l}}}{x_{i_{l}}}\right) \\
& \quad= \begin{cases}\theta_{i}(\xi) \frac{d x_{i_{1}}}{x_{i_{1}}} \wedge \cdots \wedge \frac{\widehat{d x_{i}}}{x_{i}} \wedge \cdots \wedge \frac{d x_{i_{l}}}{x_{i_{l}}} & \text { if } i \in\left\{i_{1}, \ldots, i_{l}\right\}, \\
0 & \text { otherwise, }\end{cases}
\end{aligned}
$$


whose kernel is easily seen to be $K_{B}^{*}\left(I, I^{\prime} \cup\{i\}\right)$. Thus there is a short exact sequence of complexes

$$
0 \longrightarrow K_{B}^{\cdot}\left(I, I^{\prime} \cup\{i\}\right) \longrightarrow K_{B}^{\cdot}\left(I, I^{\prime}\right) \longrightarrow \tilde{K}_{B}^{\cdot}\left(I \backslash\{i\}, I^{\prime}\right) \longrightarrow 0 .
$$

We use this exact sequence and Corollary 6.3 to compute the cohomology of each $K_{B}^{*}\left(I, I^{\prime}\right)$.

THEOREM 6.5. Suppose that $h$ is nondegenerate relative to $\Delta(h)$ and convenient relative to $S_{\mathrm{af}} \cap S_{\mathrm{sp}}$. Then

$$
\begin{aligned}
H^{l}\left(K_{B}^{*}\left(I, I^{\prime}\right), \delta_{h_{I}, \alpha}\right) & =0 \quad(l \neq|I|), \\
\operatorname{dim}_{F} H^{|I|}\left(K_{B}^{\cdot}\left(I, I^{\prime}\right), \delta_{h_{I}, \alpha}\right) & =\sum_{C \subseteq I^{\prime}}(-1)^{|C|}(|I|-|C|) ! V\left(h_{I \backslash C}\right),
\end{aligned}
$$

where $V\left(h_{I \backslash C}\right)$ denotes the volume of $\Delta\left(h_{I \backslash C}\right)$ relative to Lebesgue measure on $\mathbf{R}^{|I \backslash C|}$.

Proof. The proof is by induction on $\left|I^{\prime}\right|$. Suppose $I^{\prime}=\emptyset$. The hypothesis implies that $h_{I}$ is nondegenerate relative to $\Delta\left(h_{I}\right)$ and convenient relative to $I_{\mathrm{sp}}^{\mathrm{af}}$, so the theorem is an immediate consequence of Corollary 6.3. Now suppose the theorem is known for sets $I^{\prime}$ of a given cardinality and let $i \in I_{\mathrm{sp}}^{\mathrm{af}}, i \notin I^{\prime}$. The induction hypothesis implies that

$$
H^{l}\left(K_{B}^{\cdot}\left(I, I^{\prime}\right)\right)=H^{l}\left(\tilde{K}_{B}^{\cdot}\left(I \backslash\{i\}, I^{\prime}\right)\right)=0
$$

for $l \neq|I|$, so the long exact homology sequence associated to (6.4) shows that $H^{l}\left(K_{B}^{\cdot}\left(I, I^{\prime} \cup\{i\}\right)\right)=0$ for $l \neq|I|$ also. The exact homology sequence then gives

$$
\begin{aligned}
\operatorname{dim}_{F} H^{|I|}\left(K_{B}^{\cdot}(I,\right. & \left.\left.I^{\prime} \cup\{i\}\right)\right) \\
& =\operatorname{dim}_{F} H^{|I|}\left(K_{B}^{\cdot}\left(I, I^{\prime}\right)\right)-\operatorname{dim}_{F} H^{|I|-1}\left(K_{B}^{\cdot}\left(I \backslash\{i\}, I^{\prime}\right)\right) .
\end{aligned}
$$

The induction hypothesis gives a formula for each term on the right-hand side, and an easy calculation then gives the desired formula for the left-hand side.

Applying this in the case $I=S, I^{\prime}=S_{\text {af }} \cap S_{\text {sp }}$ gives the following.

Corollary 6.6. Suppose that $h$ is nondegenerate relative to $\Delta(h)$ and convenient relative to $S_{\mathrm{af}} \cap S_{\mathrm{sp}}$. Then

$$
\begin{aligned}
H^{l}\left(\Omega^{\cdot}(* Z), \delta_{h, \alpha}\right) & =0 \\
\operatorname{dim}_{F} H^{N+r}\left(\Omega^{\cdot}(* Z), \delta_{h, \alpha}\right) & =\sum_{C \subseteq S_{\mathrm{af}} \cap S_{\mathrm{sp}}}(-1)^{|C|}(N+r-|C|) ! V\left(h_{S \backslash C}\right) .
\end{aligned}
$$


We now assume that $h$ is given by (2.2). In section 5 it was proved that the hypothesis that $h$ be nondegenerate relative to $\Delta(h)$ and $\operatorname{dim} \Delta(h)=$ $N+r$ implies that the hypothesis of Proposition 3.1 holds. Thus under the hypothesis of Corollary 6.6 the conclusion of Proposition 3.1 holds, and by Theorem 2.8 we have the following.

THEOREM 6.7. Suppose that $\alpha_{N+1}, \ldots, \alpha_{N+r} \notin \mathbf{Z}$ and that $h=$ $\sum_{j=1}^{r} x_{N+j} f_{j}$ is nondegenerate relative to $\Delta(h)$ and convenient relative to $S_{\mathrm{af}} \cap S_{\mathrm{sp}}$. Then

$$
\begin{aligned}
H^{l}\left(\Omega^{*}(* Y), \nabla_{g, \alpha}\right) & =0 \\
\operatorname{dim}_{F} H^{N}\left(\Omega^{\cdot}(* Y), \nabla_{g, \alpha}\right) & =\sum_{C \subseteq S_{\mathrm{af}} \cap S_{\mathrm{sp}}}(-1)^{|C|}(N+r-|C|) ! V\left(h_{S \backslash C}\right) .
\end{aligned}
$$

\section{$\S 7$. An example}

In this section we apply Theorem 6.7 in the case of twisted de Rham cohomology on $\mathbf{A}^{N}$ where $g, f_{1}, \ldots, f_{r} \in F\left[x_{1}, \ldots, x_{N}\right]$ are polynomials of degrees $d_{0}, d_{1}, \ldots, d_{r}$, respectively. We assume that for $i=1, \ldots, N$, the monomial $x_{i}^{d_{j}}$ appears in $f_{j}$ (resp. in $g$ if $j=0$ ) with nonzero coefficient and that $g(0, \ldots, 0)$ and $f_{j}(0, \ldots, 0)$ for $j=1, \ldots, r$ are all nonzero. This implies in particular that $h$ is convenient relative to $S_{\mathrm{sp}}=\{1, \ldots, N\}$. Furthermore, this makes it easy to compute $V(h)$.

We regard $\Delta(h) \subseteq \mathbf{R}^{N+r}$ as fibered over $\mathbf{R}^{r}$, the last $r$ coordinates. The projection of $\Delta(h)$ on $\mathbf{R}^{r}$ is the simplex

$$
\Delta=\left\{\left(\lambda_{1}, \ldots, \lambda_{r}\right) \in \mathbf{R}^{r} \mid \lambda_{1}+\cdots+\lambda_{r} \leq 1,0 \leq \lambda_{i} \leq 1(i=1, \ldots, r)\right\} .
$$

For $\lambda=\left(\lambda_{1}, \ldots, \lambda_{r}\right) \in \Delta$, it is easily seen that the fiber of $\Delta(h)$ over $\lambda$ is the simplex in $\mathbf{R}^{N}$ with vertices at the origin and

$$
\left(\left(1-\lambda_{1}-\cdots-\lambda_{r}\right) d_{0}+\lambda_{1} d_{1}+\cdots+\lambda_{r} d_{r}\right) \mathbf{e}_{i} \quad(i=1, \ldots, N)
$$

where $\left\{\mathbf{e}_{1}, \ldots, \mathbf{e}_{N}\right\}$ is the standard basis for $\mathbf{R}^{N}$. Thus the volume of this simplex is

$$
\frac{1}{N !}\left(d_{0}-\sum_{j=1}^{r}\left(d_{0}-d_{j}\right) \lambda_{j}\right)^{N}
$$

which implies that

$$
(N+r) ! V(h)=\frac{(N+r) !}{N !} \int_{\Delta}\left(d_{0}-\sum_{j=1}^{r}\left(d_{0}-d_{j}\right) \lambda_{j}\right)^{N} d \lambda_{1} \wedge \cdots \wedge d \lambda_{r} .
$$


A straight-forward calculation using induction on $r$ then shows that the right-hand side equals the sum of all monomials of degree $N$ in $d_{0}, d_{1}, \ldots, d_{r}$, i.e.,

$$
(N+r) ! V(h)=\sum_{i_{0}+i_{1}+\cdots+i_{r}=N} d_{0}^{i_{0}} d_{1}^{i_{1}} \cdots d_{r}^{i_{r}} .
$$

We remark that this formula appeared previously in relation to the exponential sum corresponding to $h$ (see [AS1, AS2]).

Let $D_{N}\left(d_{0}, \ldots, d_{r}\right)$ denote the expression on the right-hand side of (7.1). Our hypothesis on $g, f_{1}, \ldots, f_{r}$ implies that for $I \subseteq S$ with $S_{\mathrm{du}} \subseteq I$, we have $|I| ! V\left(h_{I}\right)=D_{\left|I_{\mathrm{sp}}\right|}\left(d_{0}, \ldots, d_{r}\right)$. If we assume now that $h$ is also nondegenerate relative to $\Delta(h)$, we have the following consequence of Theorem 6.7:

$$
\begin{aligned}
H^{l}\left(\Omega^{\cdot}(* Y), \nabla_{g, \alpha}\right) & =0 \\
\operatorname{dim}_{F} H^{N}\left(\Omega^{\cdot}(* Y), \nabla_{g, \alpha}\right) & =\sum_{i=0}^{N}\left(\begin{array}{c}
N \\
i
\end{array}\right)(-1)^{N-i} D_{i}\left(d_{0}, \ldots, d_{r}\right) .
\end{aligned}
$$

When $d_{1}=\cdots=d_{r}=1$, one can check that this formula agrees with the result of $[\mathrm{AKOT}]$.

\section{REFERENCES}

[A] A. Adolphson, Hypergeometric functions and rings generated by monomials, Duke Math. J., 73 (1994), 269-290.

[AS1] A. Adolphson and S. Sperber, Newton polyhedra and the degree of the L-function associated to an exponential sum, Invent. Math., 88 (1987), 555-569.

[AS2] A. Adolphson and S. Sperber, On the degree of the L-function associated with an exponential sum, Comp. Math., 68 (1988), 125-159.

[AS3] A. Adolphson and S. Sperber, Exponential sums and Newton polyhedra: Cohomology and estimates, Ann. of Math., 130 (1989), 367-406.

[AS4] A. Adolphson and S. Sperber, Twisted exponential sums and Newton polyhedra, J. reine und angew. Math., 443 (1993), 151-177.

[AS5] A. Adolphson and S. Sperber, On the zeta function of a complete intersection, Ann. Sci. E. N. S. (to appear).

[AKOT] K. Aomoto, M. Kita, P. Orlik, and H. Terao, Twisted de Rham cohomology groups of logarithmic forms (preprint).

[B] V. V. Batyrev, Variations of the mixed Hodge structure of affine hypersurfaces in algebraic tori, Duke Math. J., 69 (1993), 349-409.

[DW1] B. Dwork, On the zeta function of a hypersurface, Publ. Math. I. H. E. S., 12 (1962), 5-68.

[DW2] B. Dwork, Generalized Hypergeometric Functions, Oxford Mathematical Monographs, Clarendon, New York, 1990. 
[DL] B. Dwork and F. Loeser, Hypergeometric series, Japan. J. Math., 19 (1993), $81-129$.

[GKZ] I. M. Gelfand, M. M. Kapranov, and A. V. Zelevinsky, Generalized Euler integrals and A-hypergeometric functions, Adv. Math., 84 (1990), 255-271.

[H] M. Hochster, Rings of invariants of tori, Cohen-Macaulay rings generated by monomials, and polytopes, Ann. of Math., 96 (1972), 318-337.

[K1] N. Katz, Thesis (1966), Princeton University.

[K2] N. Katz, On the differential equations satisfied by period matrices, Publ. Math. I. H. E. S., 35 (1968), 223-258.

[KI] M. Kita, On vanishing of the twisted rational de Rham cohomology associated with hypergeometric functions, Nagoya Math. J., 135 (1994), 55-85.

[KO] A. G. Kouchnirenko, Polyèdres de Newton et nombres de Milnor, Invent. Math., 32 (1976), 1-31.

[M] H. Matsumura, Commutative Ring Theory, Cambridge University Press, Cambridge, 1986 .

\author{
Alan Adolphson \\ Department of Mathematics \\ Oklahoma State University \\ Stillwater, Oklahoma 74078 \\ adolphs@. math.okstate.edu \\ Steven Sperber \\ School of Mathematics \\ University of Minnesota \\ Minneapolis, Minnesota 55455 \\ sperber@vx.cis.umn.edu
}

